\title{
Critical Theory of Non-Fermi Liquid Fixed Point in Multipolar Kondo Problem
}

\author{
Adarsh S. Patri® and Yong Baek Kim \\ Department of Physics and Centre for Quantum Materials, University of Toronto, \\ Toronto, Ontario M5S 1A7, Canada
}

(Received 26 May 2020; revised 31 July 2020; accepted 15 September 2020; published 29 October 2020)

\begin{abstract}
When the ground state of a localized ion is a non-Kramers doublet, such localized ions may carry multipolar moments. For example, $\operatorname{Pr}^{3+}$ ions in a cubic environment would possess quadrupolar and octupolar, but no magnetic dipole, moments. When such multipolar moments are placed in a metallic host, unusual interactions between these local moments and conduction electrons arise, in contrast to the familiar magnetic dipole interactions in the classic Kondo problem. In this work, we consider the interaction between a single quadrupolar-octupolar local moment and conduction electrons with $p$-orbital symmetry as a concrete model for the multipolar Kondo problem. We show that this model can be written most naturally in the spin-orbital entangled basis of conduction electrons. Using this basis, the perturbative renormalization-group (RG) fixed points are readily identified. There are two kinds of fixed points, one for the twochannel Kondo and the other for a novel fixed point. We investigate the nature of the novel fixed point nonperturbatively using non-Abelian bosonization, current algebra, and conformal field theory approaches. It is shown that the novel fixed point leads to a, previously unidentified, non-Fermi liquid state with entangled spin and orbital degrees of freedom, which shows resistivity $\rho \sim T^{\Delta}$ and diverging specific heat coefficient $C / T \sim T^{-1+2 \Delta}$, with $\Delta=1 / 5$. Our results open up the possibility of myriads of non-Fermi liquid states, depending on the choices of multipolar moments and conduction electron orbitals, which would be relevant for many rare-earth metallic systems.
\end{abstract}

DOI: 10.1103/PhysRevX.10.041021

\section{INTRODUCTION}

Quantum theory of metals is often discussed in the framework of Fermi liquid, where well-defined fermionic quasiparticles dominate thermodynamic and transport properties. The discovery of metallic systems that demonstrated anomalous behaviors [1,2], such as a diverging specific heat coefficient and subquadratic temperature dependence of resistivity, prompted many decades of research activities on non-Fermi liquid states. However, the number of concrete theoretical examples that are well understood is not so large. The classic Kondo problem [3-5], where the local magnetic dipole moment of a single localized ion interacts with conduction electrons' spin, is one prominent example where the dichotomy between Fermi liquid and non-Fermi liquid behaviors can be seen [6,7]. We call this the dipolar Kondo problem to clearly distinguish it from the model that we study in this work. If we consider $m$ channels of conduction electrons interacting with the local spin- $1 / 2$ moment, the

Published by the American Physical Society under the terms of the Creative Commons Attribution 4.0 International license. Further distribution of this work must maintain attribution to the author(s) and the published article's title, journal citation, and DOI.
Subject Areas: Condensed Matter Physics, Magnetism, Strongly Correlated Materials $m=1$ system is a Fermi liquid, where the local moment is screened and becomes part of the Fermi sea [8-12], while the $m>1$ systems lead to non-Fermi liquid states with anomalous properties [13-24]. This is an example where a single impurity can fundamentally change the nature of the manybody ground state.

In this work, we demonstrate the existence of a novel non-Fermi liquid state in the multipolar Kondo problem, where the local moment is characterized by a non-Kramers crystal-field doublet that carries multipolar moments such as quadrupolar or octupolar moments. While such multipolar moments are abundant in quantum materials with $f$-electron moments [25-29], the corresponding Kondo problem [30-32] has not been fully understood. Taking the example of $\mathrm{Pr}^{3+}$ ions in a cubic environment, these ions support only quadrupolar and octupolar moments and do not carry any magnetic dipole moment. If we introduce pseudospin-1/2 operators, $\mathbf{S}=\left(S^{x}, S^{y}, S^{z}\right)$ for the doublet, $S^{x}$ and $S^{y}\left(S^{z}\right)$ represent quadrupolar (octupolar) moments. While they satisfy the canonical SU(2) algebra, the physical contents of these operators are very different from the spin$1 / 2$ moment. In particular, these multipolar moments do not couple solely to conduction electron spins but rather to conduction electron bilinears that transform in the same way that the quadrupolar or octupolar moments transform. 
In order to consider a concrete model, we take $p$-orbital bands of conduction electrons, which belong to the $T_{2}$ irreducible representation of the $T_{d}$ point group in cubic systems.

We show that this model can be written most naturally if the spin-orbital entangled basis, or the total angularmomentum basis, is used for conduction electrons. This method is interesting because the conduction electrons themselves do not have any spin-orbit coupling in this model. It is the coupling to the multipolar moments that forces the conduction electrons to have strong spin-orbital entanglement. In this basis, it is shown that the perturbative RG fixed points can be easily identified. As shown earlier in the nonentangled basis [33], there exist two kinds of fixed points: one that has the behavior of the two-channel Kondo fixed point and the other being a novel fixed point. In the perturbative RG analysis [33-35], the scaling dimension of the leading irrelevant operator at the novel fixed point is $1+\Delta$, with $\Delta=1 / 4$ [33], which leads to the resistivity $\rho \sim T^{1 / 4}$ and specific heat $C \sim T^{1 / 2}$ behaviors. However, the nature of this novel fixed point was not clearly understood. In addition, the stability of this fixed point beyond the perturbative analysis was not addressed. Since the novel fixed point may represent a previously unidentified nonFermi liquid state, it is important to develop a deeper understanding of the nature of this fixed point.

Focusing on the novel fixed point, we first analyze the strong-coupling limit of the RG flow, where the coupling constants are taken to be much larger than $\mathcal{O}(1)$, and we show that this strong-coupling limit is unstable. This strongly suggests that the intermediate coupling fixed point found in the perturbative RG analysis is stable. In order to obtain nonperturbative results, we employ non-Abelian bosonization, current algebra, and conformal field theory approaches to examine the critical theory of the novel fixed point. Using the conformal embedding $[17,36]$, the free theory of conduction electrons can be written as a $\mathrm{U}(1) \times \mathrm{SU}(3)_{2} \times$ $\mathrm{SU}(2)_{3}$ Kac-Moody invariant conformal field theory. The multipolar local moment or the pseudospin- $1 / 2$ only couples to a subsector (three of eight generators) of $\mathrm{SU}(3)_{2}$. These three generators form a closed algebra. We highlight that this is not the two-channel SU(3) Kondo model, as only three generators are coupled to the multipolar local moment. It is more useful to consider the coset construction $\mathrm{SU}(3)_{2}=$ [three-state Potts model] $\times \widetilde{\mathrm{SU}}(2)_{8}$, where the multipolar local moment then couples to the $\widetilde{\mathrm{SU}}(2)_{8}$ sector. Here, $\widetilde{\mathrm{SU}}(2)_{8}$ refers to $\mathrm{SU}(2)_{8}$ with a convenient normalization of its generators. Considering the boundary conformal field theory [37,38], we find that the leading irrelevant operator at the novel fixed point is present in the $\widetilde{\mathrm{SU}}(2)_{8}$ sector. If we consider a generalized model, $\mathrm{U}(1) \times \mathrm{SU}(3)_{k} \times \mathrm{SU}(k)_{3}$, this would correspond to an operator belonging to $\mathrm{SU}(2)_{4 k}$. The scaling dimension of this operator is $1+\Delta$, with $\Delta=2 /(4 k+2)$. The perturbative fixed point corresponds to the large $k$ limit, and hence $\Delta=1 /(2 k)$. With $k=2$ (as in our case), this reproduces $1 / 4$ in the perturbative $\mathrm{RG}$ analysis. The corresponding exact scaling dimension is $\Delta=1 / 5$ for $k=2$. This leads to singular behavior for experimentally relevant quantities, such as the specific heat coefficient $C / T \sim T^{-1+2 \Delta}=T^{-3 / 5}$ and the resistivity $\rho \sim T^{\Delta}=T^{1 / 5}$. This represents a rare example of solvable non-Fermi liquid fixed points. In the broader context, our work provides a concrete example of the possibility of a wide variety of Kondo effects, as well as a myriad of non-Fermi liquids, which may arise in rare-earth metallic compounds.

\section{MICROSCOPIC MODEL}

The combination of spin-orbit (SO) coupling and crystalline electric fields (CEFs) in rare-earth compounds allows for the development of exotic higher-rank multipolar moments. Taking a localized $\mathrm{Pr}^{3+}$ ion in a cubic environment as a concrete example, the SO-coupled $J=4$ multiplet of $f^{2}$ electrons is split by the CEF to give rise to a low-lying non-Kramers $\Gamma_{3 g}$ doublet [39]. This $\Gamma_{3 g}$ doublet can support time-reversal even quadrupolar moments $\mathcal{O}_{20}=\frac{1}{2}\left(3 J_{z}^{2}-J^{2}\right)$, $\mathcal{O}_{22}=\frac{\sqrt{3}}{2}\left(J_{x}^{2}-J_{y}^{2}\right)$, as well as a time-reversal odd octupolar moment $\mathcal{T}_{x y z}=\frac{\sqrt{15}}{6} \overline{J_{x} J_{y} J_{z}}$, where the overline represents a fully symmetrized product. These multipolar moments can be efficiently described by the pseudospin-1/2 operator $\mathbf{S}=\left(S^{x}, S^{y}, S^{z}\right)$,

$S^{x}=-\frac{1}{4} \mathcal{O}_{22}, \quad S^{y}=-\frac{1}{4} \mathcal{O}_{20}, \quad S^{z}=\frac{1}{3 \sqrt{5}} \mathcal{T}_{x y z}$.

Embedding such multipolar moments in a metallic system, the localized electronic configuration can fluctuate from its $f^{2}$ ground-state configuration $\left(\Gamma_{3 g}\right)$ to excited $f^{1}$ states $\left(\Gamma_{7}\right)$ via hybridization with the sea of conduction electrons. Group theoretically, this hybridization process occurs only if the conduction electrons possess the appropriate symmetry, i.e., $\Gamma_{c}=\Gamma_{3 g} \otimes \Gamma_{7}=\Gamma_{8}$, where $\Gamma_{c}$ denotes the irrep of the conduction electron states [31]. As described in a recent work [33], one way to form this $\Gamma_{8}$ irrep is from the combination of cubic $p$-like orbitals equipped with a spinor$1 / 2$ degree of freedom, $\Gamma_{c}=p \otimes \frac{1}{2}=\Gamma_{8} \oplus \Gamma_{6}$.

The natural physical setting for such a construction is in the family of cubic rare-earth compounds, $\operatorname{Pr}(\mathrm{Ti}, \mathrm{V})_{2} \mathrm{Al}_{20}$ $\left(\operatorname{PrIr}_{2} \mathrm{Zn}_{20}\right.$ ), where the $\mathrm{Pr}^{3+}$ ions are subjected to a local $T_{d}$ symmetry by a surrounding cage of $\mathrm{Al}(\mathrm{Zn})$ atoms [40-46]. Focusing on the choice of conduction $p$ electron orbitals, the $T_{d}$ symmetry-permitting couplings of the conduction electrons to a local moment (located at impurity site $\mathbf{x}=0$ ) are

$$
\begin{aligned}
H_{1}= & K_{1}\left[S^{x}\left(c_{x, \alpha}^{\dagger} c_{x, \beta}-c_{y, \alpha}^{\dagger} c_{y, \beta}\right)\right. \\
& \left.+\frac{S^{y}}{\sqrt{3}}\left(2 c_{z, \alpha}^{\dagger} c_{z, \beta}-c_{x, \alpha}^{\dagger} c_{x, \beta}-c_{y, \alpha}^{\dagger} c_{y, \beta}\right)\right] \delta_{\alpha \beta},
\end{aligned}
$$




$$
\begin{aligned}
H_{2}= & -\sqrt{3} K_{2}\left[S^{x}\left(i \sigma_{\alpha \beta}^{x} c_{y, \alpha}^{\dagger} c_{z, \beta}-i \sigma_{\alpha \beta}^{y} c_{z, \alpha}^{\dagger} c_{x, \beta}\right)\right. \\
+ & \frac{S^{y}}{\sqrt{3}}\left(2 i \sigma_{\alpha \beta}^{z} c_{x, \alpha}^{\dagger} c_{y, \beta}-i \sigma_{\alpha \beta}^{x} c_{y, \alpha}^{\dagger} c_{z, \beta}-i \sigma_{\alpha \beta}^{y} c_{z, \alpha}^{\dagger} c_{x, \beta}\right) \\
+ & \text { H.c. }], \\
& H_{3}=K_{3} S^{z}\left[\sigma_{\alpha \beta}^{x}\left(c_{y, \alpha}^{\dagger} c_{z, \beta}+\text { H.c. }\right)+\text { cyclic }\right],
\end{aligned}
$$

where $c_{\mathcal{P}, \alpha}$ denotes the conduction electron annihilation operator at site 0 , orbital $\mathcal{P}$, and spin $\alpha$, and an implicit summation over conduction spin indices $\alpha, \beta=\{\uparrow, \downarrow\}$. We note that this construction is based on symmetries and hence is broadly applicable to cubic $\left(T_{d}\right)$ systems. We note that in terms of cubic harmonics, these terms can easily be seen as satisfying the $T_{d}$ symmetry. In particular, we note that since $S^{x}$ has an $x^{2}-y^{2}$ symmetry, it respectively couples to $x^{2}-y^{2}$ charge densities in Eq. (2) and currents in Eq. (3). We stress that in the $T_{d}$ point group, $x$ and $y z$ transform identically, and correspondingly for the cyclic permutations. Thus, Eqs. (2) and (3) have the same symmetry structure.

As studied in Ref. [33], performing third-order perturbative renormalization-group calculations leads to two nontrivial fixed points (each of these comes as a pair, which are related by a canonical transformation of the pseudospin): (i) Fixed point I: $K_{1}=-\sqrt{3} K_{2}=K_{3}=\frac{1}{2 \sqrt{3}}$; (ii) fixed point II: $K_{1}=\sqrt{12} K_{2}=-\frac{1}{2 \sqrt{6}}, K_{3}=-\frac{1}{4 \sqrt{3}}$. Fixed point I has the same exponents for physical properties from perturbative RG as the two-channel Kondo model, while fixed point II possesses highly singular scaling characterized by a leading irrelevant operator of dimension
$1+\Delta$, where $\Delta=1 / 4$ is the slope of the $\beta$ function at the fixed point. We call this the novel fixed point. The perturbative scaling can be easily understood by rewriting the $\beta$ function from Ref. [33] in terms of a single coupling constant $g_{k}$ by fixing the ratios of the original $K_{1,2,3}$ couplings to that at the fixed point of interest. For fixed point II, we can define $K_{1}=-\frac{1}{2 \sqrt{6}} g_{k}, K_{2}=-\frac{1}{12 \sqrt{2}} g_{k}$, $K_{3}=-\frac{1}{4 \sqrt{3}} g_{k}$, such that when $g_{k} \rightarrow 1$, we arrive at the fixed point II. This process leads to the $\beta$ function

$$
\frac{d g_{k}}{d \ln D}=-\frac{g_{k}^{2}}{4}+\frac{g_{k}^{3}}{4}
$$

where $D$ is the UV cutoff. The fixed point is located at $g_{k}^{*}=1$, and the slope of the $\beta$ function at the fixed point is $\Delta=1 / 4$. Indeed, this $\Delta$ appears in the leading specific heat and resistivity scaling exponents found in the original $R G$ calculation in Ref. [33].

\section{SPIN-ORBIT COUPLED BASIS FOR CONDUCTION ELECTRONS}

Though the cubic harmonics enables the symmetry nature of the coupling to be easily verified, it does not give an immediate indication as to the underlying nature of the fixed points. In order to shed light on this nature, we consider a double change of basis: (i) from cubic harmonics to spherical harmonics and (ii) to a spin-orbit coupled basis by implementation of a Clebsch-Gordon angularmomentum addition. The change of bases is delineated in Appendix A. The above Kondo couplings can be recaptured into the form

$$
\begin{aligned}
H_{\text {tot }}= & \left(-\frac{K_{1}}{\sqrt{3}}+2 K_{2}\right)\left[S^{x}\left(\left|\frac{3}{2}, \frac{1}{2}\right\rangle\left\langle\frac{3}{2}, \frac{-3}{2}|+| \frac{3}{2}, \frac{3}{2}\right\rangle\left\langle\frac{3}{2}, \frac{-1}{2}\right|+\text { H.c. }\right)\right. \\
& \left.+S^{y}\left(\left|\frac{3}{2}, \frac{-3}{2}\right\rangle\left\langle\frac{3}{2}, \frac{-3}{2}|+| \frac{3}{2}, \frac{3}{2}\right\rangle\left\langle\frac{3}{2}, \frac{3}{2}|-| \frac{3}{2}, \frac{1}{2}\right\rangle\left\langle\frac{3}{2}, \frac{1}{2}|-| \frac{3}{2}, \frac{-1}{2}\right\rangle\left\langle\frac{3}{2}, \frac{-1}{2}\right|\right)\right] \\
& +\left(\frac{\sqrt{2} K_{1}}{\sqrt{3}}+\sqrt{2} K_{2}\right)\left[S^{x}\left(\left|\frac{3}{2}, \frac{3}{2}\right\rangle\left\langle\frac{1}{2}, \frac{-1}{2}|-| \frac{3}{2}, \frac{-3}{2}\right\rangle\left\langle\frac{1}{2}, \frac{1}{2}\right|\right)+S^{y}\left(\left|\frac{3}{2}, \frac{-1}{2}\right\rangle\left\langle\frac{1}{2}, \frac{-1}{2}|-| \frac{3}{2}, \frac{1}{2}\right\rangle\left\langle\frac{1}{2}, \frac{1}{2}\right|\right)+\text { H.c. }\right] \\
& +\left(\sqrt{3} K_{3}\right) S^{z}\left[i\left|\frac{3}{2}, \frac{-3}{2}\right\rangle\left\langle\frac{3}{2}, \frac{1}{2}|+i| \frac{3}{2}, \frac{3}{2}\right\rangle\left\langle\frac{3}{2}, \frac{-1}{2}\right|+\text { H.c. }\right]
\end{aligned}
$$

where we use the ket (bra) notation of $\left|j, m_{j}\right\rangle\left(\left\langle j^{\prime}, m_{j^{\prime}}\right|\right)$ to denote conduction electron creation $c_{j, m_{j}}^{\dagger}$ (annihilation $c_{j^{\prime}, m_{j^{\prime}}}$ ) operators of total angular momentum $j\left(j^{\prime}\right)$ and $z$ projection $m_{j}\left(m_{j^{\prime}}\right)$; the impurity site location $(\mathbf{x}=0)$ is dropped for brevity. Equation (6) sheds remarkable insight into the nature of the perturbative fixed points.

\section{A. Two-channel Kondo fixed point}

First, we consider tuning of the coupling constants to fixed point I. The second term in Eq. (6) vanishes, and the remaining collection of coupling constants becomes $\left[-\left(K_{1} / \sqrt{3}\right)+2 K_{2}\right] \rightarrow 1 / 2$ and $\left(\sqrt{3} K_{3}\right) \rightarrow 1 / 2$. The remaining terms only involve the four $j=3 / 2$ states, 
which decouple into two independent (time-reversal related) doublets. Defining pseudospin-1/2 operators $\tau_{A}^{x, y, z}$ and $\tau_{B}^{x, y, z}$ for each of the decoupled doublets [Fig. 1(a): blue and purple levels, respectively], Eq. (6) can be rewritten as

$$
H_{\mathrm{tot}}=S^{x}\left(\tau_{A}^{x}+\tau_{B}^{x}\right)-S^{y}\left(\tau_{A}^{z}+\tau_{B}^{z}\right)+S^{z}\left(\tau_{A}^{y}+\tau_{B}^{y}\right),
$$

where we present the form of the $\vec{\tau}_{A, B}$ operators in Appendix B. This form is precisely that of the conventional two-channel Kondo model at its fixed point and thus confirms our perturbative determination that fixed point I has two-channel non-Fermi liquid behavior.

\section{B. Novel fixed point}

Tuning the coupling constants to the novel fixed point II, the first term in Eq. (6) vanishes, and the remaining collection of coupling constants becomes $\left[\left(\sqrt{2} K_{1} / \sqrt{3}\right)+\sqrt{2} K_{2}\right] \rightarrow$ $-1 / 4$ and $\left(\sqrt{3} K_{3}\right) \rightarrow-1 / 4$. Interestingly, the remaining terms do not belong to a single $j$ manifold but involve terms from both $j=3 / 2$ and $j=1 / 2$. This model is unlike the above two-channel model, which only involved conduction $j=3 / 2$ states. The terms can be organized into two decoupled triplets of states: (i) $\left\{\left|\frac{3}{2}, \frac{3}{2}\right\rangle,\left|\frac{3}{2}, \frac{-1}{2}\right\rangle,\left|\frac{1}{2}, \frac{-1}{2}\right\rangle\right\}$ and (ii) $\left\{\left|\frac{3}{2}, \frac{1}{2}\right\rangle,\left|\frac{3}{2}, \frac{-3}{2}\right\rangle,\left|\frac{1}{2}, \frac{1}{2}\right\rangle\right\}$, where we again use the notation of $\left|j, m_{j}\right\rangle$. Performing another unitary rotation about the $\left|\frac{1}{2}, \frac{-1}{2}\right\rangle$ axis in the (ii) space reduces the Kondo coupling to the following elegant form,

$$
H_{k}=\sum_{m=1,2} \vec{\psi}_{m}^{\dagger}(0)\left[\frac{S^{x}}{2} \frac{\lambda^{4}}{2}+\frac{S^{y}}{2} \frac{\lambda^{6}}{2}+\frac{S^{z}}{2} \frac{\lambda^{2}}{2}\right] \vec{\psi}_{m}(0),
$$

(a) Two-channel Kondo Fixed Point
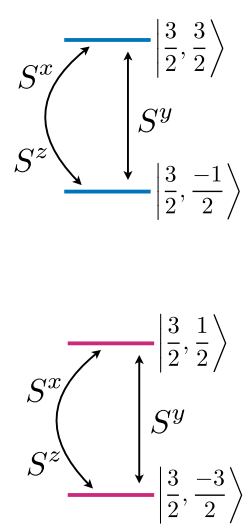

FIG. 1. Schematic picture of Kondo Hamiltonians tuned to each of the fixed points, with conduction electron transitions amongst different $\left|j, m_{j}\right\rangle$ states via coupling to the multipolar moments. (a) Two-channel Kondo fixed point, involving two decoupled pairs of conduction electrons from the $j=3 / 2$ sector (blue and purple). (b) Novel fixed point, involving two decoupled triplets of states from $j=1 / 2$ and $j=3 / 2$ conduction electron levels (orange and green). where $\lambda^{a}$ are the SU(3) Gell-Mann matrices and $m$ labels the two decoupled bases, i.e., (i) $\vec{\psi}_{m=1}^{\dagger}=\left(-c_{\frac{3}{2}, \frac{3}{2}}^{\dagger},-c_{\frac{3}{2}, \frac{1}{2}}^{\dagger}, c_{\frac{1}{2}, \frac{1}{2}}^{\dagger}\right)$ and (ii) $\vec{\psi}_{m=2}^{\dagger}=\left(c_{\frac{3}{2}, \frac{3}{2}}^{\dagger}, c_{\frac{3}{2}, \frac{1}{2}}^{\dagger}, c_{\frac{1}{2}, \frac{1}{2}}^{\dagger}\right)$, where the negative signs indicate the aforementioned final unitary transformation. Equation (8) can be schematically visualized in Fig. 1(b). Before examining the nature of this fixed point in detail, we first consider the justification of its existence from a strongcoupling limit analysis.

\section{INSTABILITY OF STRONG-COUPLING LIMIT}

The strong-coupling limit provides a consistency rationale for the existence of the perturbatively obtained fixed point. In the strong-coupling limit, the kinetic term can be ignored, and the problem reduces to determining the degeneracy of the ground state at the impurity site. If the ground state is nondegenerate, this indicates that the local moment has been quenched, and no further Kondo effects can occur. In the original Kondo model, this is the outcome, which is in agreement with the picture of a formation of a Kondo singlet. If, on the other hand, the ground state is degenerate, this indicates that, even at strong coupling, there is still enough "freedom" at the impurity site to participate in further Kondo scattering events with conduction electrons. This degeneracy thus indicates the instability of the strongly coupled fixed point, and it indicates a renormalization-group flow away from it and towards an intermediate fixed point [47].

Focusing on the novel fixed point II, the strongcoupling limit results in a fourfold degenerate ground state (Appendix C). This fourfold ground-state degeneracy is larger than the twofold degeneracy one obtains from the two-channel Kondo model, which underlines the increased quantum nature of the novel fixed point. Indeed, even with the introduction of perturbations to the strongly coupled Hamiltonian (such as conduction tunneling processes to/from the impurity), the ground state retains (at least partially) its degeneracy. Thus, even with perturbations, the strongly coupled fixed point retains sufficient degrees of freedom to scatter with conduction electrons and drive the system away from the strongly coupled fixed point, and towards an intermediate fixed point.

\section{CURRENT ALGEBRA APPROACH TO NOVEL FIXED POINT}

In the same spirit as the original Kondo problem (and even rare-earth impurity systems [31]), we consider radial (s-wave) scattering events, which allow the threedimensional free-fermion model to be mapped to a chiral one-dimensional problem [16],

$$
H_{0}=\int_{-\infty}^{\infty} d x \sum_{\beta=1,2,3 ; t=1,2} \psi_{L, \beta, t}^{\dagger}(x)\left(i \partial_{x}\right) \psi_{L, \beta, t}(x),
$$


where $x$ is the radial coordinate, $\psi_{L, \beta, j}$ denotes a leftmoving fermionic field with $\mathrm{SU}(3)$ triplet-space label $\beta, t$ is the channel index, $\beta$ sums over the respective triplet subspaces (i) and (ii) described in Sec. III B, and $v_{F}$ is the Fermi velocity, which is set to 1 . The identical Fermi velocity in Eq. (9) for all six flavors of fermions indicates an $\mathrm{SU}(3 \times 2)$ symmetry, which arises from the exact microscopic symmetries of the lattice. One can understand this symmetry by considering the conduction electron kinetic term in the original cubic harmonic ( $p$ orbitals) basis of Eqs. (2)-(4) and focusing on the Fermi surface that is well localized about the zone center [48,49]. In particular, a three-fold degeneracy of the $p_{x}, p_{y}, p_{z}$ orbitals satisfies the underlying cubic $\left(O_{h}\right)$ symmetry about this highsymmetry point, and a twofold spinful (up or down) degeneracy arises as a natural consequence of the lack of spin-orbit coupling for the (time-reversal invariant) bare conduction electrons. Translating to the spin-orbit coupled basis of Sec. III yields Eq. (9) with the SU(6) symmetry.

For definiteness, we consider the left-moving electrons as living on a circle of length $2 l$. Employing standard quantum mechanics, with the antiperiodic boundary conditions $\psi_{L}(-l)=-\psi_{L}(l)$, this case leads to (in momentum space) the allowed fermionic wave vectors $k=$ $(\pi / l)(n+1 / 2)$, where $n \in \mathbb{Z} \geq 0$. Alternatively, a $(1+1)$-dimensional free-fermion system can be described in terms of a conformal field theory. In addition, the (spatial) one dimensionality of the problem encourages one to bosonize the fermionic theory in terms of mutually commuting bosonic current operators. There are a number of different ways by which the different degrees of freedom of the fermions can be partitioned, while preserving the conformal invariance, in a procedure known as conformal embedding. The choice of the partitioning, as will become clear, depends on the problem one is considering. In any of the embeddings, only certain combinations of the states associated with the decoupled bosonic current operators can be combined so as to recover the original fermionic excitation spectrum; these combinations are called "gluing" conditions [17]. One way to decompose the above freefermion theory of $2 \times 3=6$ different flavors of fermions is in terms of its $\mathrm{U}(1)$ charge and $\mathrm{SU}(6)$ flavor degree of freedom. Here, each fermion flavor carries its own U(1) charge, $Q_{i}$, such that the total charge $Q=\sum_{i=1}^{6} Q_{i}$. The conformal embedding of rewriting Eq. (9) in terms of U(1) and $\mathrm{SU}(6)_{1}$ bosonic currents has the "gluing" condition of $Q=p(\bmod 6)$, where $p$ is the highest state of $\mathrm{SU}(6)_{1}[17]$. We note that the subscript denotes the level of the Kac-Moody (KM) algebra. However, the kinetic term and Kondo coupling suggest another conformal embedding: in terms of independent $\mathrm{U}(1)$ charge, $\mathrm{SU}(3)$ flavor, and $S U(2)$ spin degrees of freedom. We define the following left-moving non-Abelian currents in terms of the complex space-time variable $z \equiv \tau+i x$,

$$
\begin{gathered}
J(z)=\sum_{\substack{\beta=\{1,2,3\} \\
t=\{1,2\}}}: \psi_{L, \beta, t}^{\dagger} \psi_{L, \beta, t}:(z), \\
J^{a}(z)=\sum_{\substack{\alpha, \beta=\{1,2,3\} \\
t=\{1,2\}}}: \psi_{L, \alpha, t}^{\dagger}\left(\frac{\lambda_{\alpha \beta}^{a}}{2}\right) \psi_{L, \beta, t}:(z), \\
J^{A}(z)=\sum_{\substack{\beta=\{1,2,3\} \\
t, q=\{1,2\}}}: \psi_{L, \beta, t}^{\dagger}\left(\frac{\sigma_{t q}^{A}}{2}\right) \psi_{L, \beta, q}:(z),
\end{gathered}
$$

where the three currents in Eqs. (10)-(12) denote the U(1) charge, SU(3) Gell-Mann, and SU(2) spin bosonic currents, respectively; $a=\{1,2, \ldots, 8\}$ sums over the eight $\mathrm{SU}(3)$ (Gell-Mann) generators; $A=\{x, y, z\}$ sums over the three SU(2) (Pauli) generators; and “: ... :" refers to normal ordering by point splitting, i.e., $: \psi^{\dagger} \psi:(z) \equiv$ $\lim _{\delta \rightarrow 0}\left[\psi^{\dagger}(z-i \delta) \psi(z)-\left\langle\psi^{\dagger}(z-i \delta) \psi(z)\right\rangle\right]$. In effect, bosonizing the theory with these non-Abelian currents amounts to the decomposition of the irreps of the $\mathrm{SU}(6)_{1}$ in terms of irreps of $\mathrm{SU}(3)_{2} \times \mathrm{SU}(2)_{3}$. This decomposition preserves the central charge (a sufficient condition for preserving conformal invariance $[17,36]$ ) and, importantly, allows a relationship to be established between the $\mathrm{U}(1)$ charge $(Q)$ and the irrep labels for $\mathrm{SU}(3)_{2}$ and $\mathrm{SU}(2)_{3}$. We present the conformal branching rules of $\mathrm{SU}(3)_{2} \oplus \mathrm{SU}(2)_{3} \subset \mathrm{SU}(6)_{1}$ in Appendix F.

These bosonic currents satisfy the following respective $\mathrm{KM}$ algebra, which can be obtained (Appendix D) from the mode expansions of the operator product expansions (OPEs),

$$
\begin{gathered}
{\left[J_{n}, J_{m}\right]=6 n \delta_{n+m},} \\
{\left[J_{n}^{a}, J_{m}^{b}\right]=i f^{a b c} J_{n+m}^{c}+\frac{2}{2} n \delta^{a b} \delta_{n+m},} \\
{\left[J_{n}^{A}, J_{m}^{B}\right]=i \epsilon^{A B C} J_{n+m}^{C}+\frac{3}{2} n \delta^{A B} \delta_{n+m},}
\end{gathered}
$$

where we introduce the modes from the Laurent expansion $J^{a}(z)=\sum_{n \in \mathbb{Z}} z^{-n-1} J_{n}^{a}$ etc., and $f^{a b c}$ and $\epsilon^{A B C}$ are the structure constants of the $\mathrm{SU}(3)$ and $\mathrm{SU}(2)$ Lie algebras, respectively. We note that the normalization of the highest root is set to 1 (the canonical convention) to compute the above KM algebra. As seen, the SU(3) Gell-Mann and $\mathrm{SU}(2)$ spin currents satisfy $\mathrm{SU}(3)_{2}$ and $\mathrm{SU}(2)_{3} \mathrm{KM}$ algebra, respectively. Using these bosonic currents, the kinetic term can thus be recaptured in the following Sugawara form,

$$
H_{0}=\frac{1}{12}: J J:(z)+\frac{1}{5}: J^{a} J^{a}:(z)+\frac{1}{5}: J^{A} J^{A}:(z) .
$$


The free-fermion theory is a $\mathrm{U}(1) \times \mathrm{SU}(3)_{2} \times \mathrm{SU}(2)_{3} \mathrm{KM}$ invariant conformal field theory. The Kondo coupling of Eq. (8) can also be rewritten in terms of these bosonic currents,

$$
H_{K}=g_{k}\left(J^{4}(z) \frac{S^{x}}{2}+J^{6}(z) \frac{S^{y}}{2}+J^{2}(z) \frac{S^{z}}{2}\right),
$$

where we tune the ratio of the couplings such that the perturbative fixed point is reached at $g_{k}^{*}=1$.

The Sugawara form suggests that we may "complete the square" and absorb the Kondo coupling into the free Hamiltonian (with the addition of a trivial energy constant) by redefining a "shifted" bosonic current. Of course, this process can only be performed for certain special values of the Kondo coupling constant $g_{k}$ to preserve the KM algebra. In our case, when $g_{k}^{c}=2 / 5, S^{x, y, z} / 2$ can be "absorbed" into their respective $J^{4,6,2}$ currents to give the Sugawara form,

$$
\begin{aligned}
H_{0}+H_{K}= & \frac{1}{12}: J J:(z)+\frac{1}{5}: \mathcal{J}^{a} \mathcal{J}^{a}:(z) \\
& +\frac{1}{5}: J^{A} J^{A}:(z)
\end{aligned}
$$

where $\quad \mathcal{J}^{4} \equiv J^{4}+\left(S^{x} / 2\right), \quad \mathcal{J}^{6} \equiv J^{6}+\left(S^{y} / 2\right), \quad \mathcal{J}^{2} \equiv$ $J^{2}+\left(S^{z} / 2\right)$, and the remaining currents are unaffected, i.e., $\mathcal{J}^{a}=J^{a}$ for $a \neq\{2,4,6\}$, etc. Importantly, these absorbed SU(3) Gell-Mann currents satisfy the same $\mathrm{SU}(3)_{2} \mathrm{KM}$ algebra. In the same spirit as Affleck and Ludwig [23], we interpret Eq. (18) as the effective Hamiltonian and $g_{k}^{c}=2 /(k+3)=2 / 5$ as the coupling constant at the infrared (IR) fixed point. In that sense, both $\mathrm{UV}\left(g_{k}=0\right)$ and IR $\left(g_{k}^{c}=2 / 5\right)$ have the same $\mathrm{U}(1) \times$ $\mathrm{SU}(3)_{2} \times \mathrm{SU}(2)_{3}$ symmetry. Finally, we notice that the IR coupling constant $g_{k}^{c}$ reduces to the perturbative fixed point $g_{k}^{*}$ in the limit of large $k$ and then set $k \rightarrow 2$.

Indeed, the absorption of the multipolar impurity into the free electron theory is a consequence of assuming that the critical point corresponds to a conformally invariant boundary condition on the bulk conformal theory. The reasonableness of this assumption can be understood by considering the correlation function between two points far away from the impurity [23]. The impurity, we recall, is located at the origin, so we can be visualize it as the space-(imaginary) time boundary above which the free fermions and Kondo interaction exist. Now, since the points are far from the boundary, we naturally expect the correlation function to probe the bulk free-fermion (conformal) behavior. However, if the two points are closer to the boundary than to each other, it is now prudent to expect that the boundary can influence the correlation function. As such, the impurity acts as a termination of the bulk conformal theory behavior, i.e., as a boundary condition to the bulk theory. Indeed, since the impurity is fixed at $\mathbf{x}=\mathbf{0}$, under the conformal transformation, we thus expect the impurity to act as a conformally invariant boundary condition to the bulk theory. In the renormalization-group sense, the impurity is absorbed into the continuum fermion theory for increasing length scales, and its influence is retained as a modification of the free-fermion boundary condition.

Interestingly, only select SU(3) generators have absorbed the $\mathrm{SU}(2)$ impurity, and one may notice that the absorbed impurity comes with a factor of $1 / 2$. This $1 / 2$ factor is the crucial difference in this model, as it relates the $\{2-4-6\}$ subalgebra of $\mathrm{SU}(3)$ to the $\mathrm{SU}(2)$ algebra, which have structure constants of $f^{246}=1 / 2$ and $\epsilon^{x y z}=1$, respectively. The inclusion of the $1 / 2$ factor makes it possible to reimagine both the $\{2-4-6\}$ subalgebra and $\mathbf{S} / 2$ as belonging to different irreducible representations of a "1/4-quantized" SU(2) Lie algebra.

\section{1/4-QUANTIZED SU(2) LIE ALGEBRA}

To understand this algebra, we define the generators of the algebra as $T_{2,4,6}$ such that they satisfy $\left[T_{2}, T_{4}\right]=$ $(i / 2) T_{6}$, in any representation. Drawing an analogy to typical $\mathrm{SU}(2), T_{6}$ is analogous to $\sigma^{z} / 2$, so we define the highest $T_{6}$ state $|\tilde{j}\rangle$ such that $T_{6}|\tilde{j}\rangle=\tilde{j}|\tilde{j}\rangle$. In addition, we define raising or lowering operators $T^{ \pm}$, which raise and lower the $T_{6}$ eigenvalue by $\pm 1 / 2$ (as shown in Appendix E). In the same spirit as SU(2), the existence of a "lowest" state constrains the allowed values of $\tilde{j}$ to be $\tilde{j}=\ell / 4$, where $\ell \in \mathbb{R}$; we refer to $\tilde{j}$ as "physical-spin" labels. In the more formal language of Lie algebras, the physical-spin label is related to the Dynkin labels, $\tilde{j}=\tilde{\lambda}_{1} / 4$ (as shown in Appendix E). The $\tilde{j}=1 / 4$ representation is a two-dimensional representation with $T_{2}=\left(\sigma^{x} / 4\right), T_{4}=$ $\left(\sigma^{y} / 4\right), T_{6}=\left(\sigma^{z} / 4\right)$, which is precisely $\mathbf{S} / 2$. The $\tilde{j}=1 / 2$ representation is a three-dimensional representation with $T_{2}=\left(\lambda^{2} / 2\right), T_{4}=\left(\lambda^{4} / 2\right), T_{6}=\left(\lambda^{6} / 2\right)$ (this representation is used, up to unitary transformation, in the Kondo coupling). Finally, the quadratic Casimir for a given $\tilde{j}$ representation is $Q=\tilde{j}(\tilde{j}+1 / 2) \mathbb{I}$.

To understand the relation of the subalgebra to the typical SU(2) Lie algebra, we impose a generic normalization condition by fixing the length of the longest root $\left|\alpha_{1}^{2}\right|=\eta / 4$, where $\eta \in \mathbb{R}>0$ is a chosen normalization convention. Since the roots are the weights of the adjoint representation, the introduction of the $\eta$ normalization factor amounts to modification of the structure constants and, subsequently, a redefinition of the generators. In particular, the redefined generators of SU(3), $\bar{T}_{i} \equiv \sqrt{\eta} T_{i}$, satisfy the Lie algebra $\left[\bar{T}_{i}, \bar{T}_{j}\right]=\sqrt{\eta} f_{i j}^{k} \bar{T}_{k}$, where $T_{i}$ and $f_{i j}^{k}$ are the canonically normalized generators and structure constants of SU(3) used in constructing the Sugawara form. This redefinition of the generators results in a modification of the OPE of SU(3) currents, 


$$
\overline{\boldsymbol{J}}^{a}\left(z_{1}\right) \bar{J}^{b}\left(z_{2}\right) \sim \frac{1}{\left(z_{12}\right)^{2}} \tilde{k} \delta^{a b}+\frac{1}{z_{12}} i \sqrt{\eta} f^{a b c} \overline{\boldsymbol{J}}^{c},
$$

where $\tilde{k} \equiv(\eta k / 2)$ and $z_{12} \equiv z_{1}-z_{2}$, and the overline indicates current operators redefined to obey the generalized normalization. We note that $k=2$ always. We can now notice that for different choices of the normalization, we can map the subalgebra to "more familiar" (canonically normalized) Lie algebras. In the original $\eta=1$ convention, we have the canonically normalized $\{2-4-6\}$ subalgebra of $\mathrm{SU}(3)$. For $\eta=4$, the subalgebra gets mapped to the canonically normalized SU(2) Lie algebra. We can thus see that for a particular choice of the normalization, we have a one-to-one correspondence between the original $\{2-4-6\}$ subalgebra and $\mathrm{SU}(2)$.

With this generalized-normalization formulation, the affine extension of the subalgebra can thus be easily constructed in analogous methods to the conventional $\mathrm{SU}(2)$ case. Because of the embedding of the $\{2-4-6\}$ subalgebra in the larger affine $\mathrm{SU}(3)_{2}$ algebra, the level of the embedded subalgebra is given by $(8 \tilde{k} / n)=4 k$. This embedded level restricts the highest physical-spin state to be $\tilde{j} \leq k$. As a consequence, the fusion coefficients can be similarly constructed and are of the form

$$
\tilde{j}_{1} \otimes \tilde{j}_{2}=\bigoplus_{\tilde{j}_{3}} \mathcal{N}_{\tilde{j}_{1}, \tilde{j}_{2}}^{(4 k) \tilde{j}_{3}},
$$

where

$$
\mathcal{N}_{\tilde{j}_{1}, \tilde{j}_{2}}^{(4 k) \tilde{j}_{3}}=\left\{\begin{array}{l}
1 \text { if }\left|\tilde{j}_{1}-\tilde{j}_{2}\right| \leq \tilde{j}_{3} \leq \min \left(\tilde{j}_{1}+\tilde{j}_{2}, \frac{2(4 k)}{4}-\tilde{j}_{1}-\tilde{j}_{2}\right) \\
0 \text { otherwise, }
\end{array}\right.
$$

where we return back to the normalization of $\eta=1$. The fusion coefficients become an important ingredient in determining the modification of the free-fermion boundary conditions due to the absorption of the multipolar impurity.

\section{MAVERICK COSET CONSTRUCTION: THREE-STATE POTTS MODEL}

The full Hilbert space of the free electron model involves $\mathrm{U}(1)$ charge, $\mathrm{SU}(3)_{2}$ flavor, and $\mathrm{SU}(2)_{3}$ spin degrees of freedom. The symmetry group of the flavor degree of freedom is governed by $\mathcal{G}=\mathrm{SU}(3)_{2}$ and involves currents $J_{\mathcal{G}}^{a}$; the subscript $\mathcal{G}$ explicitly recalls that these currents belong to the complete $\mathrm{SU}(3)_{2}$ group. However, as seen in Eq. (17), only a select number of the $\mathrm{SU}(3)_{2}$ bosonic currents couple to the local moment, suggesting that an alternative conformal embedding would be helpful when considering the Kondo interaction. To proceed, we denote the subgroup $\mathcal{H} \in \mathcal{G}$, with currents $J_{\mathcal{H}}^{a}$ that belong to the $\{2-4-6\}$ sector, and construct stress-energy tensors belonging to each of the groups,

$$
\begin{aligned}
T_{\mathcal{G}}(z) & =\frac{1 / 2}{\tilde{k}+C_{A, \mathcal{G}} / 2} \sum_{a=1}^{8}: J_{\mathcal{G}}^{a}(z) J_{\mathcal{G}}^{a}(z):, \\
T_{\mathcal{H}}(z) & =\frac{1 / 2}{\tilde{k}+C_{A, \mathcal{H}} / 2} \sum_{a=1}^{3}: J_{\mathcal{H}}^{a}(z) J_{\mathcal{H}}^{a}(z):,
\end{aligned}
$$

where $\tilde{k}=\eta k / 2$ just as before, and $C_{A}$ are the quadratic Casimirs of the adjoint representation of the respective groups, i.e., $C_{A, \mathcal{G}}=3 \eta$ and $C_{A, \mathcal{H}}=\eta / 2$. The conformal weight (or dimension) of the primary states of a representation $\tilde{j}$ of $\mathcal{H}$ is given by

$$
h_{\tilde{j}}=\frac{C_{\tilde{j}} / 2}{\tilde{k}+C_{A, \mathcal{H}} / 2}=\frac{\frac{\eta}{2} \tilde{j}(\tilde{j}+1 / 2)}{\frac{\eta k}{2}+\frac{\eta}{4}}=\frac{\tilde{j}(\tilde{j}+1 / 2)}{k+1 / 2},
$$

where in the final quality, we notice that the normalization dependencies of the quadratic Casimirs and $\tilde{k}$ drop out in the conformal weight (or dimension). Though the conformal weight is independent of the normalization, we notice that for $\eta=4$ [the normalization condition to map the $\{2-4-6\}$ subalgebra to canonical $\mathrm{SU}(2)]$, the conformal weight of the primary states is identical to that of $\mathrm{SU}(2)_{8}$, as expected.

The procedure of breaking up the stress-energy tensors is attractive. This is because, though the currents $T_{\mathcal{G}}(z)$ and $T_{\mathcal{H}}(z)$ have singular contributions in their OPE with $J_{\mathcal{H}}^{a}$ individually, the OPE of $T_{\mathcal{G} / \mathcal{H}} \equiv T_{\mathcal{G}}-T_{\mathcal{H}}$ with $J_{\mathcal{H}}^{a}$ is non singular and, subsequently, so is the OPE of $T_{\mathcal{G} / \mathcal{H}}$ with all of $T_{\mathcal{H}}$ [50]. As such, the Virasoro algebra generated by $T_{\mathcal{G}}$ can be decomposed into two mutually commuting Virasoro algebras, $\left[T_{\mathcal{G} / \mathcal{H}}, T_{\mathcal{H}}\right]=0$. This formulation, known as a coset construction, permits the efficient "breaking up" of a larger group into smaller subgroups. To understand the nature of the coset, $\mathcal{G} / \mathcal{H}$, we consider its central charge,

$c_{\mathcal{G} / \mathcal{H}}=c_{\mathcal{G}}-c_{\mathcal{H}}=\frac{\tilde{k} \operatorname{dim}|\mathcal{G}|}{\tilde{k}+C_{A, \mathcal{G}} / 2}-\frac{\tilde{k} \operatorname{dim}|\mathcal{H}|}{\tilde{k}+C_{A, \mathcal{H}} / 2}=\frac{4}{5}$,

where $\operatorname{dim}|\mathcal{G}|=8$ and $\operatorname{dim}|\mathcal{H}|=3$. The central charge is independent of the normalization convention, as can be easily seen by both $\tilde{k}$ and $C_{A}$ scaling linearly in $\eta$. The central charge of $4 / 5$ corresponds to the three-state Potts model, in addition to the minimal $\mathcal{M}(6,5)$ model. Inspired by the normalization of $\eta=4$ resulting in mapping the $\{2-4-6\}$ subalgebra to the canonically normalized $\mathrm{SU}(2)$ algebra of level $4 k=8$, we consider this coset model to be described by the maverick coset formulation $\mathrm{SU}(3)_{2} / \mathrm{SU}(2)_{8}[51,52]$.

Employing this coset formulation, the IR Hamiltonian in Eq. (18) can be reimagined as a $\mathrm{U}(1) \times$ (three-state Potts model $) \times \widetilde{\mathrm{SU}}(2)_{8} \times \mathrm{SU}(2)_{3} \mathrm{KM}$ invariant conformal field 
theory; the $\sim$ denotes $1 / 4$ quantization of the $\mathrm{SU}(2)_{8} \mathrm{KM}$ algebra. In order to make this association, we need the socalled branching functions, which act as further conditions to describe which irreps of the parent $\mathrm{SU}(3)_{2}$ are associated with which primary field of the three-state Potts model and which irrep of $\mathrm{SU}(2)_{8}$. We list the branching functions of this maverick coset model and the field associations in Appendix G. We highlight that only four of the (three-state Potts model) primary fields enter into our identifications: the "identity" field $(\mathbb{I})$, the thermal operator field $(\epsilon)$, the spin operator field $(\sigma)$, and the $\mathcal{Z}$ field, with corresponding conformal weight (or dimension) of $0,2 / 5,1 / 15$, and $2 / 3$, respectively.

\section{FINITE-SIZE SPECTRUM}

The above-formulated theory is appropriately prepared for the application of boundary conformal field theory (B-CFT) of Cardy [37,38] and an extension of the approach as applied by Affleck and Ludwig [16,23]. The essence of Cardy's B-CFT [37,38] is that it avoids handling of complicated boundary conditions and instead focuses on boundary states; Appendix I briefly describes this relationship. These boundary states are related to the multiplicity coefficients $n_{A B}^{\alpha}$, which determine the spectrum of excitations for particular boundary conditions. In our context, $\alpha=\left(Q, j_{f}\right.$, [three-state Potts model field], $\left.\tilde{j}\right)$, where each label denotes the highest state of the respective algebra, and the nontrivial boundary condition $(B)$ is due to having a single multipolar impurity at the origin. The corresponding nontrivial boundary states can be obtained from known (free-fermion, $A$ ) boundary states by the elegant "fusion rule" hypothesis of Affleck and Ludwig [16,23], whereby the fusion coefficients of Eq. (20) relate the multiplicity coefficients of different boundary conditions. Since the impurity only couples to the $\widetilde{\mathrm{SU}}(2)_{8}$ irreps $(\tilde{j}$ quantum number), the fusion is performed within the $\widetilde{\mathrm{SU}}(2)_{8}$ sector only, leaving the other irrep labels unchanged, i.e., $n_{A B}^{\tilde{j}}=$ $\sum_{\tilde{j}_{2}} \mathcal{N}_{\tilde{j}_{2}, \tilde{j}_{I}}^{\tilde{j}} n_{A A}^{\tilde{j}_{2}}$, where we take $\tilde{j}_{I}=1 / 4$, as the impurity spin belongs to the $\tilde{j}=1 / 4$ representation.

One of the key features of B-CFT is the existence of boundary operators with nontrivial conformal dimensions. These operators give rise to singular contributions to the free energy and, subsequently, to the various response functions of interest. Under the conformal mapping of a semi-infinite plane ( $x-t$ half-plane) with a particular choice of boundary conditions to a finite strip, the primary boundary operators with boundary condition $B$ on the plane are in a one-to-one correspondence to the states of the finite strip with boundary conditions $B B$ (both ends of the strip) [23]. Most importantly, the energies of the states in the finite-strip conformal tower are the scaling dimensions of the boundary operators. In our context, this amounts to considering the free-fermion tower and performing a double fusion so as to obtain the appropriate scaling dimensions of the boundary operators. Indeed, it is the lowest-lying state (after the double fusion) that gives rise to the leading irrelevant boundary operator.

Employing the conformal embedding [which relates the $\mathrm{U}(1)$ charge $Q$ to the irreps of flavor, $\mathrm{SU}(3)_{2}$, and spin, $\mathrm{SU}(2)_{3}$ ] and the coset branching rules [which relates the irreps of $\mathrm{SU}(3)_{2}$ to the coset fields and $\widetilde{\mathrm{SU}}(2)_{8}$ irreps], the energy of a primary state is given,

$E_{\mathrm{tot}}=\frac{\pi}{l}\left[\frac{Q^{2}}{12}+\frac{j_{f}\left(j_{f}+1\right)}{3+2}+h_{\mathrm{coset}}+\frac{\tilde{j}(\tilde{j}+1 / 2)}{2+1 / 2}\right]$,

where $h_{\text {coset }}$ is the scaling dimension of the coset primary field (Appendix G), $j_{f}$ labels the irrep of the $\mathrm{SU}(2)_{3}$ flavor degree of freedom, and $\tilde{j}$ denotes the $1 / 4$-quantized $\mathrm{SU}(2)_{8}$ degree of freedom. Table I lists the finite-size energy spectrum for the free-fermion states, as given by Eq. (26).

The impact of the multipolar impurity is accounted for by the double fusion $\left(n_{B B}^{\tilde{j}}=\sum_{\tilde{j}_{2}, \tilde{j}_{3}} \mathcal{N}_{\tilde{j}_{2}, \tilde{j}_{I}}^{\tilde{j}} \mathcal{N}_{\tilde{j}_{3}, \tilde{j}_{I}}^{\tilde{j}_{2}} n_{A A}^{\tilde{j}_{3}}\right)$ with the free-fermion $\tilde{j}$ states to yield the conformal tower in Table II. Under the double fusion, the "spin" $\tilde{j}$ label changes to $0 \rightarrow 0, \frac{1}{2} ; \frac{1}{2} \rightarrow 0, \frac{1}{2}, 1 ; 1 \rightarrow \frac{1}{2}, 1, \frac{3}{2}$; etc. We emphasize that only the "reshuffling" of the 1/4-quantized $\mathrm{SU}(2)_{8}$ states takes place, with the irreps of the other degrees of freedom remaining unaffected. The lowest nonzero energy state corresponds to a primary state that is chargeless $(Q=0)$, flavorless $\left(j_{f}=0\right)$, coset-trivial $(\mathbb{I})$, and of $1 / 4$-quantized "spin" $\tilde{j}=1 / 2$. The scaling dimension of the corresponding boundary operator $\vec{\phi}$ is $\Delta=1 / 5$.

Any operator that enters into the fixed-point Hamiltonian must preserve the symmetry of the $\mathrm{U}(1) \times$ [three-state Potts model $] \times \widetilde{\mathrm{SU}}(2)_{8} \times \mathrm{SU}(2)_{3} \mathrm{KM}$ invariance of the conformal field theory. On physical grounds, we also

TABLE I. Free-fermion tower with antiperiodic boundary conditions at $x=l$. The employed primary fields of the threestate Potts model are $\mathbb{I}, \epsilon, \sigma$, and $\mathcal{Z}$. We note that we only consider the primary states here, and we present states with energies $E_{\mathrm{tot}} \leq \pi / l$; the remaining primary states are given in Appendix $\mathrm{H}$.

\begin{tabular}{|c|c|c|c|c|}
\hline$Q, \mathrm{U}(1)$ & $j_{f}, \mathrm{SU}(2)_{3}$ & $\begin{array}{l}\text { [Three-state } \\
\text { Potts model] }\end{array}$ & $\tilde{j}, \widetilde{\mathrm{SU}}(2)_{8}$ & $(l / \pi) E_{\mathrm{tot}}$ \\
\hline 0 & 0 & $\mathbb{I}$ & 0 & 0 \\
\hline 1 & $\frac{1}{2}$ & $\sigma$ & $\frac{1}{2}$ & $\frac{1}{2}$ \\
\hline 0 & 1 & $\mathbb{I}$ & 1 & 1 \\
\hline 0 & 1 & $\epsilon$ & $\frac{1}{2}$ & 1 \\
\hline 2 & 0 & $\sigma$ & 1 & 1 \\
\hline 2 & 0 & $\mathcal{Z}$ & 0 & 1 \\
\hline 2 & 1 & $\sigma$ & $\frac{1}{2}$ & 1 \\
\hline$\vdots$ & $\vdots$ & $\vdots$ & $\vdots$ & $\vdots$ \\
\hline
\end{tabular}


TABLE II. Primary operator content after double fusion of the free-fermion tower in Table I, with multipolar impurity at $x=0$ and $x=l$. The employed primary fields of the three-state Potts model are $\mathbb{I}, \epsilon, \sigma$, and $\mathcal{Z}$. Only the primary states of energies $E_{\text {tot }} \leq \pi / l$ are listed here; the remaining states are given in Appendix H.

\begin{tabular}{|c|c|c|c|c|}
\hline$Q, \mathrm{U}(1)$ & $j_{f}, \mathrm{SU}(2)_{3}$ & $\begin{array}{l}\text { [Three-state } \\
\text { Potts model] }\end{array}$ & $\tilde{j}, \widetilde{\mathrm{SU}}(2)_{8}$ & $(l / \pi) E_{\mathrm{tot}}$ \\
\hline 0 & 0 & $\mathbb{I}$ & 0 & 0 \\
\hline 0 & 0 & $\mathbb{I}$ & $\frac{1}{2}$ & $\frac{1}{5}$ \\
\hline 1 & $\frac{1}{2}$ & $\sigma$ & 0 & $\frac{3}{10}$ \\
\hline 1 & $\frac{1}{2}$ & $\sigma$ & $\frac{1}{2}$ & $\frac{1}{2}$ \\
\hline 0 & 1 & $\mathbb{I}$ & $\frac{1}{2}$ & $\frac{3}{5}$ \\
\hline 2 & 0 & $\sigma$ & $\frac{1}{2}$ & $\frac{3}{5}$ \\
\hline 0 & 1 & $\epsilon$ & 0 & $\frac{4}{5}$ \\
\hline 2 & 1 & $\sigma$ & 0 & $\frac{4}{5}$ \\
\hline 1 & $\frac{1}{2}$ & $\sigma$ & 1 & $\frac{9}{10}$ \\
\hline 0 & 1 & $\mathbb{I}$ & 1 & 1 \\
\hline 0 & 1 & $\epsilon$ & $\frac{1}{2}$ & 1 \\
\hline 2 & 0 & $\mathcal{Z}$ & 0 & 1 \\
\hline 2 & 0 & $\sigma$ & 1 & 1 \\
\hline 2 & 1 & $\sigma$ & $\frac{1}{2}$ & 1 \\
\hline$\vdots$ & $\vdots$ & $\vdots$ & $\vdots$ & $\vdots$ \\
\hline
\end{tabular}

expect that the leading irrelevant operator will involve the primary "spin" field $\vec{\phi}$, as the Kondo coupling occurs between the multipolar impurity and the conduction electrons occur in the 1/4-quantized "spin" sector. In order to meet the symmetry requirement, we can simply consider the application of a KM current density operator $\mathcal{J}_{n<0}^{a}$ (where $a=\{2,4,6\}$ ), which generates descendent states/ operators when acted on a primary state/operator. Thus, the candidate for the leading irrelevant operator (that obeys the KM symmetry) is the first-descendent operator $\overrightarrow{\mathcal{J}}_{-1} \cdot \vec{\phi}$, which is explicitly a "spinless" object (as it is a scalar product of two "spin" operators) and has a scaling dimension of $1+\Delta$.

\section{PHYSICAL PROPERTIES: SPECIFIC HEAT, RESISTIVITY, AND ENTROPY}

To characterize the novel fixed point, we first consider its impact on the thermodynamic and transport response functions such as specific heat and resistivity. As detailed by Affleck and Ludwig [16], the leading irrelevant operator plays a central role in determining the scaling behavior of these response functions. In particular, the fixed-point Hamiltonian, and subsequently free energy, is augmented by the leading irrelevant operator at the boundary $\left(\delta g_{k} \overrightarrow{\mathcal{J}}_{-1} \cdot \vec{\phi}\right)$, from which the specific heat is computed from second-order perturbation theory in $\delta g_{k}$. This process yields the specific heat coefficient scaling as $C / T \sim$ $T^{-1+2 \Delta}=T^{-3 / 5}$. Similarly, the leading-order corrections to the scattering rate (due to the impurity) are computed by perturbing the one-electron Green's function linearly by the leading irrelevant operator [19]. The subsequent correction to the resistivity scales as $\rho \sim T^{\Delta}=T^{1 / 5}$. The coefficient of this correction is $\delta g_{k}$, so the sign of the deviation is determined by whether the intermediate fixed point is approached from above $\left(\delta g_{k}>0\right)$ or below $\left(\delta g_{k}<0\right)$.

Finally, the residual entropy $\mathcal{S}_{\text {imp }}(T)$, which provides a measure for the ground-state degeneracy of the novel fixed point, can be computed via the modular S-matrix [19,20] (as described in Appendix J). The entropy is found to be $\mathcal{S}_{\text {imp }}(T=0)=\ln \{[\sin (\pi / 5)] /[\sin (\pi / 10)]\} \approx 0.643$, which is just under twice the residual entropy of the two-channel Kondo model fixed point.

\section{DISCUSSIONS}

In this work, we elucidated the nature of the novel nonFermi liquid fixed point in the multipolar Kondo problem by employing non-Abelian bosonization, current algebra, and boundary conformal field theory approaches. A crucial finding of our work is that the most natural language to express the multipolar Kondo couplings is in the spin-orbital entangled $\left|j, m_{j}\right\rangle$ basis. This finding suggests that though conduction electrons may have spin and orbital quantum numbers to begin with, their interaction with a single multipolar forces the two decoupled degrees of freedom to become intertwined. This provides a route to controllably introduce spin-orbital entangling in a metallic system with the incorporation of multipolar impurities.

From the finite-size spectrum, the scaling behavior of experimentally relevant quantities associated with the fixed point is obtained nonperturbatively; in particular, the specific heat coefficient $C / T \sim T^{-1+2 \Delta}=T^{-3 / 5}$, and the resistivity $\rho \sim T^{\Delta}=T^{1 / 5}$. In addition, the residual entropy $\mathcal{S}_{\text {imp }}(T=$ $0) \approx 0.643$ is almost twice that of the two-channel Kondo model [20]. The highly singular nature of the response functions concretely establishes the non-Fermi liquid nature of the novel fixed point. We emphasize that though the scaling behavior of the leading irrelevant operator looks the same as in the eight-channel dipolar Kondo model, the nature of the fixed point is different due to the other conformal sectors. More specifically, the complete finite-size spectrum is distinct in the novel fixed point, which can be tested by future numerical renormalization-group computations.

An important requirement in realizing such an exotic Kondo effect is to ensure that the symmetry-protected degeneracies are preserved. Breaking the local $T_{d}$ symmetry (leading to splitting of the non-Kramers doublet ground state) or lifting the cubic symmetry of the underlying lattice (breaking the degeneracy of the conduction electron bands) by an external perturbation results in the demise of this Kondo problem, as the Kondo interactions describe energy-preserving scattering events. In effect, symmetry-breaking perturbations are relevant in the renormalization-group sense for the 
nontrivial fixed point. This can be understood from more general considerations where one examines the influence of a field $\left(h_{\phi}\right)$ conjugate to a boundary operator $(\phi)$ with scaling dimension $\Delta_{\phi}$. The associated term added to the Lagrangian is $\delta \mathcal{L}\left(h_{\phi}\right)=h_{\phi} \int d \tau \phi(\tau)$, where the spatial integral is absent as the boundary operator is confined to $\mathbf{x}=\mathbf{0}$. Under the standard renormalization-group procedure $\left(\tau \rightarrow \tau^{\prime}=\tau / b\right.$, where $b>1$ ), the conjugate field scales as $b^{1-\Delta_{\phi}}$. The field conjugate to the primary boundary operator is thus relevant in the renormalization-group sense. Physically, this conjugate field can be associated as lattice-stress fields that couple to the multipolar impurity [31] and split the non-Kramers degeneracy. We note that a symmetry-preserving perturbation, such as hydrostatic pressure or chemical pressure by substitution of the transition metal in the $\operatorname{Pr}(\mathrm{TM})_{2} \mathrm{Al}_{20}$, acts as an irrelevant perturbation to the nontrivial fixed point.

The solvable problem tackled in this work provides the foundation for a diverse variety of Kondo effects and nonFermi liquids. In particular, though our work is motivated by the $\operatorname{Pr}(\mathrm{Ti}, \mathrm{V})_{2} \mathrm{Al}_{20}$ family and as such focuses on $\mathcal{O}_{20}, \mathcal{O}_{22}$ quadrupolar, and $\mathcal{T}_{x y z}$ octupolar local moments, this is just one out of a myriad of possibilities. Indeed, there are examples of other $4 f[53-56]$ and $5 f[57-61,61]$ electrons subjected to noncubic crystalline electric fields, which give rise to different possible combinations of supported multipolar moments. In addition, conduction electrons may arrive with their own diversity in their orbital degrees of freedom, beyond the cubic $p$ considered in this work $[48,49]$. The combination of these two sources of diversity suggests that many different Kondo effects may occur, which could lead to a multitude of non-Fermi liquid behaviors. This study thus opens a new route and territory for achieving and studying exotic Kondo effects and novel non-Fermi liquids.

In terms of future work, a thorough classification of the possible non-Fermi liquids that may occur in multipolarbased compounds would be an intriguing and impactful study. In addition, the extension of the single-impurity problem to the corresponding multipolar Kondo lattice problem $[62,63]$ and associated quantum critical phenomena are outstanding questions for future research.

\section{ACKNOWLEDGMENTS}

We thank Piers Coleman and Sungjay Lee for helpful discussions. This work was supported by NSERC of Canada. Y. B. K. is supported by the Killam Research Fellowship of the Canada Council for the Arts.

\section{APPENDIX A: CUBIC HARMONICS TO SPIN-ORBITAL ENTANGLED BASIS}

In order to rewrite the Kondo coupling in terms of the spin-orbital entangled $j, m_{j}$ basis, we need to perform a double change of basis. First, the cubic harmonics are rewritten in terms of $L=1$ orbital angular-momentum degrees of freedom,

$$
\begin{gathered}
\left|p_{x}\right\rangle=\frac{1}{\sqrt{2}}(|1,-1\rangle-|1,1\rangle)_{L}, \\
\left|p_{y}\right\rangle=\frac{i}{\sqrt{2}}(|1,-1\rangle+|1,1\rangle)_{L}, \\
\left|p_{z}\right\rangle=|1,0\rangle_{L},
\end{gathered}
$$

where we use the basis $\left|L, m_{L}\right\rangle$ for the angular-momentum orbital degrees of freedom (the subscript $L$ is a perpetual remainder). Second, the spin and orbital degrees of freedom are entangled by angular-momentum addition via ClebschGordon coefficients,

$$
\begin{aligned}
\left|1,1 ; \frac{1}{2}, \uparrow\right\rangle & =\left|\frac{3}{2}, \frac{3}{2}\right\rangle_{J}, \\
\left|1,1 ; \frac{1}{2}, \downarrow\right\rangle & =\frac{1}{\sqrt{3}}\left(\left|\frac{3}{2}, \frac{1}{2}\right\rangle_{J}+\sqrt{2}\left|\frac{1}{2}, \frac{1}{2}\right\rangle_{J}\right), \\
\left|1,0 ; \frac{1}{2}, \uparrow\right\rangle & =\frac{1}{\sqrt{3}}\left(\sqrt{2}\left|\frac{3}{2}, \frac{1}{2}\right\rangle_{J}-\left|\frac{1}{2}, \frac{1}{2}\right\rangle_{J}\right), \\
\left|1,0 ; \frac{1}{2}, \downarrow\right\rangle & =\frac{1}{\sqrt{3}}\left(\sqrt{2}\left|\frac{3}{2}, \frac{-1}{2}\right\rangle_{J}+\left|\frac{1}{2}, \frac{-1}{2}\right\rangle_{J}\right), \\
\left|1,-1 ; \frac{1}{2}, \uparrow\right\rangle & =\frac{1}{\sqrt{3}}\left(\left|\frac{3}{2}, \frac{-1}{2}\right\rangle_{J}-\sqrt{2}\left|\frac{1}{2}, \frac{-1}{2}\right\rangle_{J}\right), \\
\left|1,-1 ; \frac{1}{2}, \downarrow\right\rangle & =\left|\frac{3}{2}, \frac{-3}{2}\right\rangle_{J},
\end{aligned}
$$

where, on the left-hand side of the equality, we use the notation of $\left|L, m_{L} ; s, s^{z}\right\rangle$, where $s$ is the conduction electron spin degree of freedom; on the right-hand side of the equality, we use the notation for the spin-orbital entangled basis $\left|J, m_{J}\right\rangle_{J}$, where the subscript is a perpetual remainder of the $J$ basis. In the main text, we drop this subscript as the additional clarification is not required.

\section{APPENDIX B: BASIS FOR TWO-CHANNEL KONDO FIXED POINT}

Tuning the couplings to fixed point I, only the $j=3 / 2$ conduction electron states survive. The two decoupled pseudospin- $1 / 2$ operators in the $\left|\frac{3}{2}, m_{j}\right\rangle$ space are

$$
\begin{aligned}
\tau_{A}^{x} & =\frac{1}{2}\left|\frac{3}{2}, \frac{3}{2}\right\rangle\left\langle\frac{3}{2},-\frac{1}{2}\left|+\frac{1}{2}\right| \frac{3}{2},-\frac{1}{2}\right\rangle\left\langle\frac{3}{2}, \frac{3}{2}\right|, \\
\tau_{A}^{y} & =\frac{-i}{2}\left|\frac{3}{2},-\frac{1}{2}\right\rangle\left\langle\frac{3}{2}, \frac{3}{2}\left|+\frac{i}{2}\right| \frac{3}{2}, \frac{3}{2}\right\rangle\left\langle\frac{3}{2},-\frac{1}{2}\right|, \\
\tau_{A}^{z} & =\frac{1}{2}\left|\frac{3}{2},-\frac{1}{2}\right\rangle\left\langle\frac{3}{2},-\frac{1}{2}\left|-\frac{1}{2}\right| \frac{3}{2}, \frac{3}{2}\right\rangle\left\langle\frac{3}{2}, \frac{3}{2}\right|,
\end{aligned}
$$


and its partner $\tau_{B}^{x, y, z}:\left.\tau_{A}^{x, y, z}\right|_{m_{j} \rightarrow-m_{j}}$,

$$
\begin{aligned}
& \tau_{B}^{x}=\frac{1}{2}\left|\frac{3}{2},-\frac{3}{2}\right\rangle\left\langle\frac{3}{2}, \frac{1}{2}\left|+\frac{1}{2}\right| \frac{3}{2}, \frac{1}{2}\right\rangle\left\langle\frac{3}{2},-\frac{3}{2}\right|, \\
& \tau_{B}^{y}=\frac{-i}{2}\left|\frac{3}{2}, \frac{1}{2}\right\rangle\left\langle\frac{3}{2},-\frac{3}{2}\left|+\frac{i}{2}\right| \frac{3}{2},-\frac{3}{2}\right\rangle\left\langle\frac{3}{2}, \frac{1}{2}\right|, \\
& \tau_{B}^{z}=\frac{1}{2}\left|\frac{3}{2}, \frac{1}{2}\right\rangle\left\langle\frac{3}{2}, \frac{1}{2}\left|-\frac{1}{2}\right| \frac{3}{2},-\frac{3}{2}\right\rangle\left\langle\frac{3}{2},-\frac{3}{2}\right| .
\end{aligned}
$$

\section{APPENDIX C: DEGENERACY OF STRONGLY COUPLED FIXED POINT}

The strong-coupling limit provides a means to verify the consistency of the existence of the perturbatively obtained fixed point. In the case of a single coupling constant of the original isotropic Kondo problem, this limit is unique. However, in the case of multiple couplings (as in our model), there is an inherent ambiguity as each of the coupling constants can be taken to infinity independently. To circumvent this issue, we take the reasonable and elegant approach of Nozieres and Blandin [47], where one takes each of the couplings to infinity while fixing their ratio to be that at the fixed point. This approach is the simplest extension of the strong-coupling limit of the isotropic Kondo model, while at the same time accounting for the properties of the nontrivial fixed point by fixing the ratio.

The ground state of the strongly coupled novel fixed point is fourfold degenerate,

$$
\begin{aligned}
& \left|\mathrm{GS}_{1}\right\rangle=\frac{1}{\sqrt{3}}\left[|\uparrow\rangle_{f}\left|\frac{1}{2}, \frac{-1}{2}\right\rangle+|\downarrow\rangle_{f}\left(\left|\frac{3}{2}, \frac{3}{2}\right\rangle+i\left|\frac{3}{2}, \frac{-1}{2}\right\rangle\right)\right], \\
& \left|\mathrm{GS}_{2}\right\rangle=\frac{1}{\sqrt{3}}\left[|\uparrow\rangle_{f}\left(\left|\frac{3}{2}, \frac{3}{2}\right\rangle-i\left|\frac{3}{2}, \frac{-1}{2}\right\rangle\right)+|\downarrow\rangle_{f}\left|\frac{1}{2}, \frac{-1}{2}\right\rangle\right], \\
& \left|\mathrm{GS}_{3}\right\rangle=\frac{1}{\sqrt{3}}\left[|\uparrow\rangle_{f}\left(\left|\frac{3}{2}, \frac{-3}{2}\right\rangle-i\left|\frac{3}{2}, \frac{1}{2}\right\rangle\right)-|\downarrow\rangle_{f}\left|\frac{1}{2}, \frac{1}{2}\right\rangle\right], \\
& \left|\mathrm{GS}_{4}\right\rangle=\frac{1}{\sqrt{3}}\left[|\uparrow\rangle_{f}\left|\frac{1}{2}, \frac{1}{2}\right\rangle-|\downarrow\rangle_{f}\left(\left|\frac{3}{2}, \frac{-3}{2}\right\rangle+i\left|\frac{3}{2}, \frac{1}{2}\right\rangle\right)\right],
\end{aligned}
$$

where $\left|\mathrm{GS}_{1,2}\right\rangle$ and $\left|\mathrm{GS}_{3,4}\right\rangle$, respectively, belong to the (i) and (ii) decoupled SU(3) sectors defined in Sec. III B. Note that $\left|\mathrm{GS}_{1}\right\rangle\left(\left|\mathrm{GS}_{2}\right\rangle\right)$ is related to $\left|\mathrm{GS}_{3}\right\rangle\left(\left|\mathrm{GS}_{4}\right\rangle\right)$ by timereversal symmetry. The instability of the strongly coupled fixed point can be further highlighted by placing the impurity on a one-dimensional line (parallel to the $\hat{\mathbf{x}}$ direction) and allowing tunneling of conduction electrons to the nearest neighboring sites on either side of the impurity. The tunneling acts as a perturbation to the strongly coupled Kondo Hamiltonian. We find that a twofold degenerate ground state remains at the impurity site (with up to second nearest-neighbor hopping), where one of the ground states is an equal superposition of $\left|\mathrm{GS}_{1,2}\right\rangle$, while the other is an equal superposition of $\left|\mathrm{GS}_{3,4}\right\rangle$.

\section{APPENDIX D: OPERATOR PRODUCT EXPANSION OF NON-ABELIAN CURRENTS}

The OPEs of the non-Abelian currents are crucial in determining the $\mathrm{KM}$ algebra as well as for rewriting the free-fermion Hamiltonian in terms of non-Abelian bosonic currents (Sugawara form) [64]. The OPEs for the U(1) charge, $\mathrm{SU}(3)$ current, and $\mathrm{SU}(2)$ currents are

$$
\begin{aligned}
& J\left(z_{1}\right) J\left(z_{2}\right)= \frac{6}{z_{12}^{2}}+: \psi_{L, \alpha, p}^{\dagger} \psi_{L, \alpha, p} \psi_{L, \beta, q}^{\dagger} \psi_{L, \beta, q}:\left(z_{2}\right) \\
&+ {\left[:\left(\partial_{z} \psi_{L, \alpha, p}^{\dagger}\right) \psi_{L, \alpha, p}:\right.} \\
&\left.-: \psi_{L, \alpha, p}^{\dagger}\left(\partial_{z} \psi_{L, \alpha, p}\right):\right]\left(z_{2}\right), \\
& J^{a}\left(z_{1}\right) J^{b}\left(z_{2}\right)= \frac{2}{2 z_{12}^{2}} \delta^{a b}+\frac{i f^{a b c}}{z_{12}} J^{c}\left(z_{2}\right) \\
&+\frac{1}{4} f^{a b c}: \partial_{z}\left(\psi_{L, \alpha, p}^{\dagger} \lambda_{\alpha \beta}^{c} \psi_{L, \beta, p}\right):\left(z_{2}\right) \\
&+\frac{1}{4}: \psi_{L, \alpha, p}^{\dagger} \psi_{L, \beta, p} \psi_{L, \gamma, q}^{\dagger} \psi_{L, \delta, q}:\left(z_{2}\right) \lambda_{\alpha \beta}^{a} \lambda_{\gamma \delta}^{b} \\
&+\frac{1}{4}\left(\frac{2}{3} \delta_{\alpha \beta}^{a b}+d^{a b c} \lambda_{\alpha \beta}^{c}\right)\left[:\left(\partial_{z} \psi_{L, \alpha, p}^{\dagger}\right) \psi_{L, \beta, p}:\right. \\
&\left.-: \psi_{L, \alpha, p}^{\dagger}\left(\partial_{z} \psi_{L, \beta, p}\right):\right]\left(z_{2}\right), \\
& \frac{3}{2 z_{12}^{2}} \delta^{a b}+\frac{i \epsilon^{A B C}}{z_{12}} J^{C}\left(z_{2}\right) \\
&+\frac{1}{4} \epsilon^{A B C}: \partial_{z}\left(\psi_{L, \alpha, p}^{\dagger} \sigma_{p q}^{C} \psi_{L, \alpha, q}\right):\left(z_{2}\right) \\
&+\frac{1}{4}: \psi_{L, \alpha, p}^{\dagger} \psi_{L, \alpha, q} \psi_{L, \beta, s}^{\dagger} \psi_{L, \beta, t}:\left(z_{2}\right) \sigma_{p q}^{A} \sigma_{s t}^{B} \\
&+\frac{1}{4}\left(\delta_{p q}^{a b}\right)\left[:\left(\partial_{z} \psi_{L, \alpha, p}^{\dagger}\right) \psi_{L, \alpha, q}:\right. \\
&\left.-: \psi_{L, \alpha, p}^{\dagger}\left(\partial_{z} \psi_{L, \alpha, q}\right):\right]\left(z_{2}\right), \\
& J^{A}\left(z_{1}\right) J^{B}\left(z_{2}\right)
\end{aligned}
$$

where we take $z_{1} \rightarrow z_{2}$ in the OPEs, $z_{12} \equiv z_{1}-z_{2}$, repeated indices $\{\alpha, \beta, \gamma, \delta\}=\{1,2,3\}, c=\{1,2,3\}, C=\{x, y, z\}$, $\{p, q, s, t\}=\{1,2\}$ are summed over. The level of each KM algebra can thus be read off directly from the numerator of the first term in each OPE. We note that we use the canonical normalization of the structure constants to compute the OPEs.

\section{APPENDIX E: 1/4-QUANTIZED SU(2) LIE ALGEBRA: DYNKIN LABELS AND PHYSICAL-SPIN WEIGHT}

The 1/4-quantized SU(2) Lie algebra is analogous to SU (2), and as such, we focus on some of the key differences (and draw analogies, when applicable) from SU(2). As described in the main text, we define raising or lowering operators $T^{ \pm} \equiv\left(T_{2} \pm i T_{4}\right) / \sqrt{2}$, which satisfy 


$$
\left[T_{6}, T^{ \pm}\right]= \pm \frac{1}{2} T^{ \pm}, \quad\left[T^{+}, T^{-}\right]=\frac{1}{2} T_{6} .
$$

The actions of these operators on the eigenstates of $T_{6}$ are

$$
\begin{gathered}
T_{6}|\tilde{j}, m\rangle=m|\tilde{j}, m\rangle, \\
T^{-}|\tilde{j}, m\rangle=N_{m}|\tilde{j}, m-1 / 2\rangle, \\
T^{+}|\tilde{j}, m-1 / 2\rangle=N_{m}|\tilde{j}, m\rangle,
\end{gathered}
$$

where $N_{m}=\frac{1}{2 \sqrt{2}} \sqrt{(2 \tilde{j}+2 m)(2 \tilde{j}-2 m+1)}$. Thus, we can notice that, similarly to $\mathrm{SU}(2)$, there is one label for the highest state $(\tilde{j})$ and one ladder to ascend and descend with $T^{ \pm}$.

To apply the machinery of (affine) Lie algebra, it is helpful to recapture the above notation in a more formal setting. In particular, a given representation is denoted by the highest weight (or state) $\tilde{\lambda}$, which can written in the basis of fundamental weights, $\omega_{1}=\alpha_{1} / 2$, with integer coefficients $\left(\tilde{\lambda}_{1}\right)$ known as Dynkin labels, $\tilde{\lambda}=\tilde{\lambda}_{1} \omega_{1}$. The Dynkin labels are the eigenvalues of the Chevalley basis, and the relation between the Dynkin labels and the physical-spin weight is given by $\tilde{j}=\tilde{\lambda}_{1} / 4$. This can be seen by considering the typical Lie algebra bases. In the Chevalley basis, the commutation relation is given by

$$
[e, f]=h, \quad[h, e]=2 e, \quad[h, f]=-2 f,
$$

where the Cartan matrix is the same as that of the canonical $\mathrm{SU}(2), A=(2)$. The eigenvalues of the Chevalley generator $h$ are the Dynkin labels,

$$
h|\tilde{\lambda}\rangle=\tilde{\lambda}_{1}|\tilde{\lambda}\rangle .
$$

Taking a generic normalization factor ( $\eta$ just as in the main text) for the highest root, we have the corresponding generators in the Cartan-Weyl basis,

$$
H=\frac{\sqrt{\eta}}{4} h, \quad E^{+}=e, \quad E^{-}=f,
$$

which satisfy the commutation relations $\left[E^{+}, E^{-}\right]=$ $(4 / \sqrt{\eta}) H$ and $\left[H, E^{ \pm}\right]= \pm(\sqrt{\eta} / 2) E^{ \pm}$. Finally, we have the "physical" normalization-dependent generators, $\bar{T}^{ \pm}=$ $\sqrt{(\eta / 8)} E^{ \pm}$and $\bar{T}_{6}=H$, which satisfy the commutation relations in Eq. (E1) with normalization dependency $\sqrt{\eta}$ on the right-hand side. Thus, the eigenvalue of $\bar{T}_{6}$ is given by

$$
\bar{T}_{6}|\tilde{\lambda}\rangle=H|\tilde{\lambda}\rangle=\frac{\sqrt{n} h}{4}|\tilde{\lambda}\rangle=\frac{\sqrt{n} \tilde{\lambda}_{1}}{4}|\tilde{\lambda}\rangle
$$

For $\eta=1$, we notice that $\tilde{j}=\tilde{\lambda}_{1} / 4$, which gives us the interpretation of the $\{2-4-6\}$ subalgebra as $1 / 4$ quantized. Similarly, for $\eta=4$, the algebra satisfies the "canonically normalized" SU(2) Lie algebra, with the physical weight (or label) $\tilde{\lambda}_{1} / 2$.

\section{APPENDIX F: CONFORMAL EMBEDDING: $\mathbf{S U}(3)_{2} \oplus \mathbf{S U}(2)_{3} \subset \mathbf{S U}(6)_{1}$}

Branching rules provide the decomposition coefficients of an irrep of a (affine) Lie algebra $g\left(\hat{g}_{k}\right)$ into the irreps of a smaller (affine) Lie algebra $p \subset g\left(\hat{p}_{k^{\prime}} \subset \hat{g}_{k}\right)$. In our context, we are interested in the decomposition of the irreps of $\mathrm{SU}(6)_{1}$ into $\mathrm{SU}(2)_{3} \oplus \mathrm{SU}(3)_{2}$. Since the level of $\mathrm{SU}(6)$ is 1 , the only dominant highest-weight representations are the fundamental representations $\hat{\omega}_{0,1, \ldots, 5}$. Following the procedure detailed in Chap. 17.A of Ref. [36] for employing Young tableaux and outer automorphisms, we obtain the following decomposition in Eq. (F1),

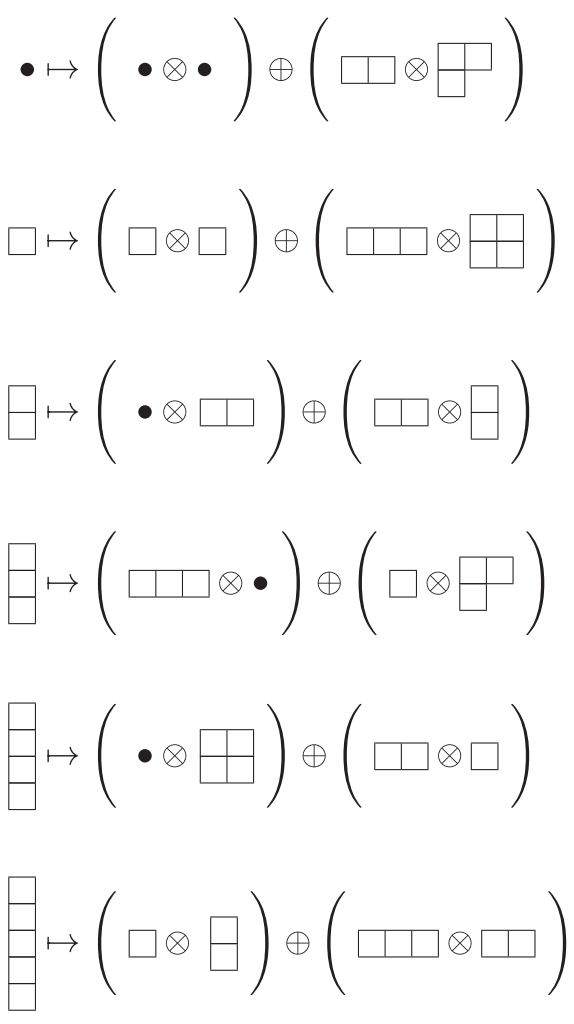

where on the right-hand side, the first (second) Young tableau labels irreps of $\mathrm{SU}(2)_{3}\left[\mathrm{SU}(3)_{2}\right]$. We can rewrite the above branching rules in terms of the Dynkin labels of the corresponding irreps, namely,

$$
\begin{aligned}
& \hat{\omega}_{0} \mapsto([3,0] \otimes[2,0,0]) \oplus([1,2] \otimes[0,1,1]), \\
& \hat{\omega}_{1} \mapsto([2,1] \otimes[1,1,0]) \oplus([0,3] \otimes[0,0,2]), \\
& \hat{\omega}_{2} \mapsto([3,0] \otimes[0,2,0]) \oplus([1,2] \otimes[1,0,1]), \\
& \hat{\omega}_{3} \mapsto([0,3] \otimes[2,0,0]) \oplus([2,1] \otimes[0,1,1]), \\
& \hat{\omega}_{4} \mapsto([3,0] \otimes[0,0,2]) \oplus([1,2] \otimes[1,1,0]), \\
& \hat{\omega}_{5} \mapsto([2,1] \otimes[1,0,1]) \oplus([0,3] \otimes[0,2,0]),
\end{aligned}
$$


where we use the standard definition of the irreps of $\mathrm{SU}(\mathrm{N})$ in terms of Dynkin labels: $\hat{\lambda}=\left[\lambda_{0}, \lambda_{1}, \ldots\right]=$ $\left[k-\sum_{i}^{r} \lambda_{i}, \lambda_{1}, \ldots\right]$, where $r$ is the rank of the Lie algebra.

\section{APPENDIX G: MAVERICK COSET BRANCHING RULES}

The spirit of the coset branching rules is analogous to that of Appendix $\mathrm{F}$ in that we once again are considering decompositions of the various representations $\hat{\lambda}$ of the affine Lie algebra $\hat{g}$ into the representations $\hat{\mu}$ of the affine Lie algebra $\hat{p}$, which is given by

$$
\hat{\lambda} \mapsto \bigoplus_{\hat{\mu}} b_{\hat{\lambda} \hat{\mu}} \hat{\mu}
$$

where $b_{\hat{\lambda} \hat{\mu}}$ are the branching coefficients. This decomposition can be rewritten in terms of characters,

$$
\chi_{\hat{\lambda}}=\sum_{\hat{\mu}} \chi_{\{\hat{\lambda} ; \hat{\mu}\}} \chi_{\hat{\mu}},
$$

where $\chi_{\hat{\lambda}} \equiv q^{h_{\hat{\lambda}}-c / 24} t r_{\hat{\lambda}}\left(q^{L_{0}}\right)$. The term in the trace accounts for the "grade" or descendent level $n$ of a state belonging to the $\hat{\lambda}$ irrep (i.e., already extracted out the conformal weight of the primary state, $h_{\hat{\lambda}}$ ). For the maverick coset, the restricted character decompositions have been carefully computed in Refs. [51,52], which we reproduce in Table III with our notations. Below each of the restricted characters in Table III, we write down the combination of the threestate Potts model characters (described below) that gives these restricted characters of the coset field. These combinations allow the conformal dimension of the coset model to be obtained. We note that the conformal dimension of the coset model is related to the conformal dimensions of the parent algebra $(\hat{g})$ and the subalgebra $(\hat{p}): h_{\chi_{\{\hat{\lambda} \hat{x} \hat{j}\}}}=$ $h_{\hat{\lambda}}+n-h_{\hat{\mu}}$; as such, $n$ can be extracted. For reference, the characters of the three-state Potts models [36] employed in Table III are

$$
\begin{aligned}
& \chi_{1,1}^{\mathrm{vir}}=q^{\frac{-1}{30}}\left(1+q^{2}+q^{3}+2 q^{4}+2 q^{5}+4 q^{6}+\ldots\right), \\
& \chi_{2,1}^{\mathrm{vir}}=q^{\frac{2}{5}-\frac{1}{30}}\left(1+q+q^{2}+2 q^{3}+3 q^{4}+4 q^{5}+6 q^{6}+\ldots\right), \\
& \chi_{3,1}^{\mathrm{vir}}=q^{\frac{7}{5}-\frac{1}{30}}\left(1+q+2 q^{2}+2 q^{3}+4 q^{4}+5 q^{5}+8 q^{6}+\ldots\right), \\
& \chi_{1,3}^{\mathrm{vir}}=q^{\frac{2}{3}-\frac{1}{30}}\left(1+q+2 q^{2}+2 q^{3}+4 q^{4}+5 q^{5}+8 q^{6}+\ldots\right), \\
& \chi_{4,1}^{\mathrm{vir}}=q^{3-\frac{1}{30}}\left(1+q+2 q^{2}+3 q^{3}+4 q^{4}+5 q^{5}+8 q^{6}+\ldots\right), \\
& \chi_{2,3}^{\mathrm{vir}}=q^{\frac{1}{15}-\frac{1}{30}}\left(1+q+2 q^{2}+3 q^{3}+5 q^{4}+7 q^{5}+10 q^{6}+\ldots\right) .
\end{aligned}
$$

\section{APPENDIX H: EXCITED STATES OF CONFORMAL TOWERS}

In the main text, we present the conformal towers for energies $E_{\text {tot }} \leq(\pi / l)$. In Tables IV and V, we present the remaining, higher energy, states for the free fermion and after the double fusion with the impurity, respectively.

TABLE III. Maverick coset $\mathrm{SU}(3)_{2} / \mathrm{SU}(2)_{8}$ branching functions as computed in Refs. [51,52]. The grade denotes the descendent level of the irreps of $\mathrm{SU}(3)_{2}$. The minimal model field labeling is the same as that in Ref. [36].

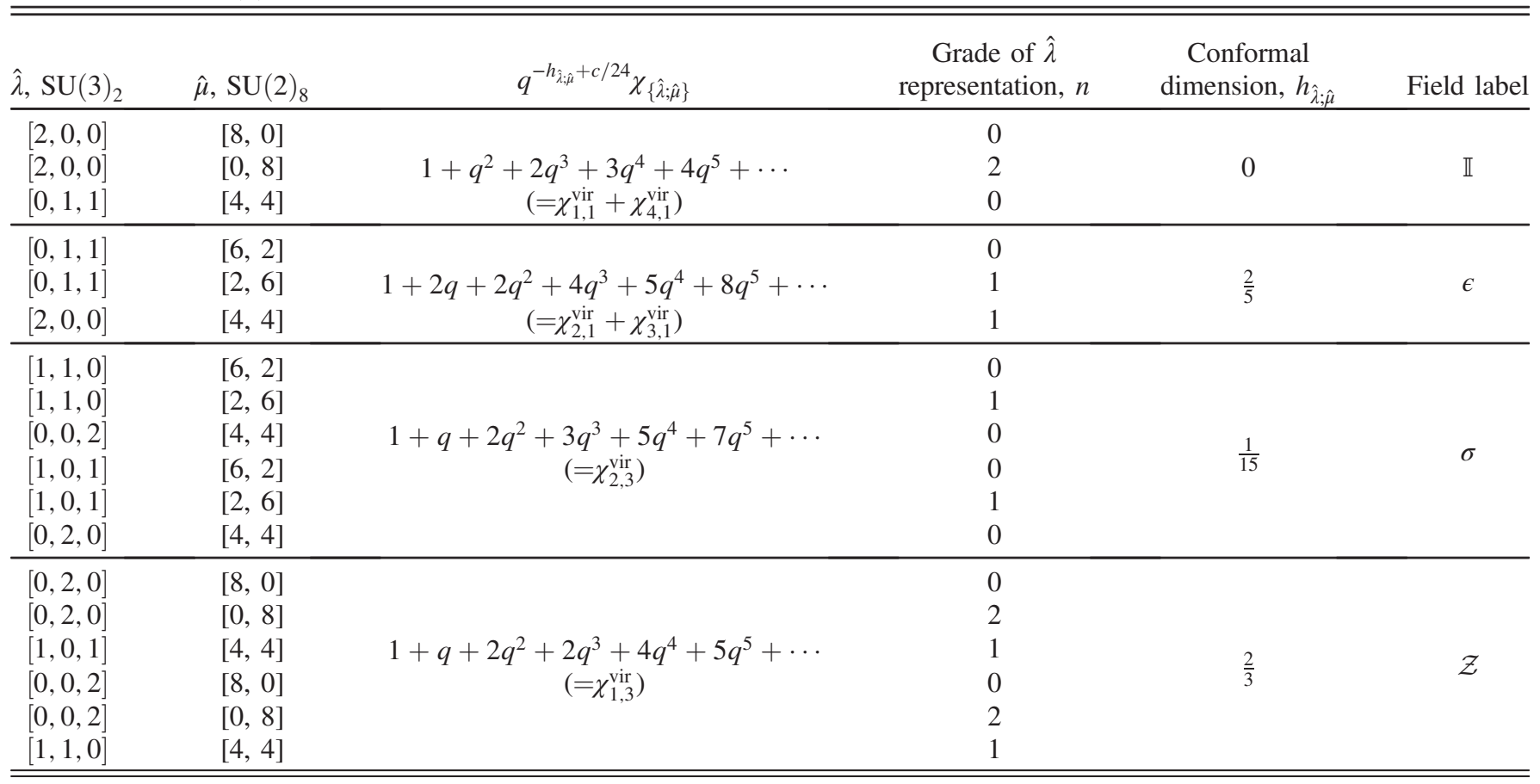


TABLE IV. Free-fermion tower with antiperiodic boundary conditions at $x=l$. The primary states for $E_{\text {tot }}>\pi / l$ are presented here; primary states with $E_{\mathrm{tot}} \leq \pi / l$ are given in Table I.

\begin{tabular}{|c|c|c|c|c|}
\hline$Q, \mathrm{U}(1)$ & $j_{f}, \mathrm{SU}(2)_{3}$ & $\begin{array}{l}\text { [Three-state } \\
\text { Potts model] }\end{array}$ & $\tilde{j}, \widetilde{\mathrm{SU}}(2)_{8}$ & $(l / \pi) E_{\mathrm{tot}}$ \\
\hline$\vdots$ & : & $\vdots$ & $\vdots$ & $\vdots$ \\
\hline 1 & $\frac{3}{2}$ & $\sigma$ & 1 & $\frac{3}{2}$ \\
\hline 1 & $\frac{3}{2}$ & $\mathcal{Z}$ & 0 & $\frac{3}{2}$ \\
\hline 3 & $\frac{3}{2}$ & $\mathbb{I}$ & 0 & $\frac{3}{2}$ \\
\hline 3 & $\frac{1}{2}$ & $\mathbb{I}$ & 1 & $\frac{3}{2}$ \\
\hline 3 & $\frac{1}{2}$ & $\epsilon$ & $\frac{1}{2}$ & $\frac{3}{2}$ \\
\hline 4 & 1 & $\sigma$ & $\frac{1}{2}$ & 2 \\
\hline 4 & 0 & $\sigma$ & 1 & 2 \\
\hline 4 & 0 & $\mathcal{Z}$ & 0 & 2 \\
\hline 5 & $\frac{1}{2}$ & $\sigma$ & $\frac{1}{2}$ & $\frac{5}{2}$ \\
\hline 5 & $\frac{3}{2}$ & $\sigma$ & 1 & $\frac{7}{2}$ \\
\hline 5 & $\frac{3}{2}$ & $\mathcal{Z}$ & 0 & $\frac{7}{2}$ \\
\hline
\end{tabular}

\section{APPENDIX I: BOUNDARY CONFORMAL FIELD THEORY AND RESIDUAL ENTROPY}

In this section, we briefly describe the rationale behind Cardy's boundary conformal field theory. This discussion is employed in calculating the residual entropy in Appendix J. We direct the reader to Refs. [23,36-38] for detailed discussions.

As described in the main text, the impurity acts as a conformally invariant boundary condition to the bulk conformal theory. In the spirit of Cardy [37,38], it is helpful to consider the conformal system on a cylinder of spatial length $l$ and periodic temporal length $\beta$ that winds around the circumference. The partition function for this system is then given by

$$
\mathcal{Z}_{A B}=\operatorname{tr}\left[\exp \left(-\beta H_{A B}\right)\right]=\sum_{a} n_{A B}^{a} \chi_{a}\left(e^{-(\pi \beta / l)}\right),
$$

where the subscript on the Hamiltonian indicates the boundary conditions in the spatial direction, $n_{A B}^{a}$ are the multiplicity coefficients described in the main text, and $\chi_{a}\left(e^{-(\pi \beta / l)}\right)$ are the characters of the Virasoro algebra for the conformal tower $a$. Equation (I1) can be understood as the quantum mechanical partition function computed over the "thermally coherent" time $\beta=1 / T$.

However, one can perform a modular transformation, which physically interchanges the temporal and spatial axes $\tau \leftrightarrow x$. The partition function for this system is

$$
\mathcal{Z}_{A B}^{\tau \leftrightarrow x}=\left\langle A\left|\exp \left(-l H_{S}\right)\right| B\right\rangle=\sum_{a}\langle A \mid a\rangle\langle a \mid B\rangle \chi_{a}\left(e^{-(4 \pi l / \beta)}\right),
$$

TABLE V. Primary operator content after double fusion of primary states of energies $E_{\text {tot }}>\pi / l$. The primary states with

\begin{tabular}{|c|c|c|c|c|}
\hline$Q, \mathrm{U}(1)$ & $j_{f}, \mathrm{SU}(2)_{3}$ & $\begin{array}{l}\text { [Three-state } \\
\text { Potts model] }\end{array}$ & $\tilde{j}, \widetilde{\mathrm{SU}}(2)_{8}$ & $(l / \pi) E_{\mathrm{tot}}$ \\
\hline$\vdots$ & $\vdots$ & $\vdots$ & $\vdots$ & $\vdots$ \\
\hline 1 & $\frac{3}{2}$ & $\sigma$ & $\frac{1}{2}$ & $\frac{11}{10}$ \\
\hline 3 & $\frac{1}{2}$ & $\mathbb{I}$ & $\frac{1}{2}$ & $\frac{11}{10}$ \\
\hline 2 & 0 & $\mathcal{Z}$ & $\frac{1}{2}$ & $\frac{6}{5}$ \\
\hline 3 & $\frac{1}{2}$ & $\epsilon$ & 0 & $\frac{5}{10}$ \\
\hline 2 & 1 & $\sigma$ & 1 & $\frac{7}{5}$ \\
\hline 0 & 1 & $\epsilon$ & 1 & $\frac{7}{5}$ \\
\hline 1 & $\frac{3}{2}$ & $\sigma$ & 1 & $\frac{3}{2}$ \\
\hline 1 & $\frac{3}{2}$ & $\mathcal{Z}$ & 0 & $\frac{3}{2}$ \\
\hline 3 & $\frac{2}{2}$ & $\mathbb{I}$ & 0 & $\frac{2}{2}$ \\
\hline 3 & $\frac{1}{2}$ & $\mathbb{I}$ & 1 & $\frac{2}{2}$ \\
\hline 3 & $\frac{1}{2}$ & $\epsilon$ & $\frac{1}{2}$ & $\frac{3}{2}$ \\
\hline 0 & 1 & $\mathbb{I}$ & $\frac{3}{2}$ & $\frac{8}{5}$ \\
\hline 2 & 0 & $\sigma$ & $\frac{3}{2}$ & $\frac{8}{5}$ \\
\hline 4 & 0 & $\sigma$ & $\frac{1}{2}$ & $\frac{8}{5}$ \\
\hline 1 & $\frac{3}{2}$ & $\mathcal{Z}$ & $\frac{1}{2}$ & $\frac{17}{10}$ \\
\hline 3 & $\frac{3}{2}$ & $\mathbb{I}$ & $\frac{1}{2}$ & $\frac{17}{10}$ \\
\hline 4 & 1 & $\sigma$ & 0 & $\frac{9}{5}$ \\
\hline 3 & $\frac{1}{2}$ & $\epsilon$ & 1 & $\frac{19}{10}$ \\
\hline 4 & 0 & $\sigma$ & 1 & 2 \\
\hline 4 & 1 & $\sigma$ & $\frac{1}{2}$ & 2 \\
\hline 4 & 0 & $\mathcal{Z}$ & 0 & 2 \\
\hline 1 & $\frac{3}{2}$ & $\sigma$ & $\frac{3}{2}$ & $\frac{21}{10}$ \\
\hline 3 & $\frac{1}{2}$ & $\mathbb{I}$ & $\frac{3}{2}$ & $\frac{21}{10}$ \\
\hline 4 & 0 & $\mathcal{Z}$ & $\frac{1}{2}$ & $\frac{11}{5}$ \\
\hline 5 & $\frac{1}{2}$ & $\sigma$ & 0 & $\frac{23}{10}$ \\
\hline 4 & 1 & $\sigma$ & 1 & $\frac{12}{5}$ \\
\hline 5 & $\frac{1}{2}$ & $\sigma$ & $\frac{1}{2}$ & $\frac{5}{2}$ \\
\hline 4 & 0 & $\sigma$ & $\frac{3}{2}$ & $\frac{13}{5}$ \\
\hline 5 & $\frac{1}{2}$ & $\sigma$ & 1 & $\frac{29}{10}$ \\
\hline 5 & $\frac{3}{2}$ & $\sigma$ & $\frac{1}{2}$ & $\frac{31}{10}$ \\
\hline 5 & $\frac{3}{2}$ & $\sigma$ & 1 & $\frac{7}{2}$ \\
\hline 5 & $\frac{3}{2}$ & $\mathcal{Z}$ & 0 & $\frac{7}{2}$ \\
\hline 5 & $\frac{3}{2}$ & $\mathcal{Z}$ & $\frac{1}{2}$ & $\frac{37}{10}$ \\
\hline 5 & $\frac{3}{2}$ & $\sigma$ & $\frac{3}{2}$ & $\frac{41}{10}$ \\
\hline
\end{tabular}
$E_{\text {tot }} \leq \pi / l$ are given in Table II.

where the subscript $S$ indicates the modular transformation has been made, and $|a\rangle$ symbolically denotes the so-called "Ishibashi" states that are used to enforce no momentum or energy flow across the boundary. Because of the interchange of the temporal and spatial axes, Eq. (I2) can be understood as the propagation of the evolution operator over "time" $l$ between initial and final states $A$ and $B$. The Virasoro characters in Eqs. (I1) and (I2) are related by the modular S-matrix, which allows one to find a relationship between the boundary conditions (encoded in the multiplicity coefficients $n_{A B}^{a}$ ) and the boundary states $(|A, B\rangle)$, 


$$
\sum_{b} S_{b}^{a} n_{A B}^{b}=\langle A \mid a\rangle\langle a \mid B\rangle
$$

where $S_{b}^{a}$ is the modular S-matrix, and $b$ sums over the conformal towers. Equation (I3) is known as "Cardy's equations," which allow boundary conditions (left-hand side) to be related to boundary states (right-hand side) [23].

\section{APPENDIX J: RESIDUAL ENTROPY}

The impurity entropy is defined as [23]

$$
\mathcal{S}_{\text {imp }}(T) \equiv \lim _{l \rightarrow \infty}\left[\mathcal{S}(l, T)-\mathcal{S}_{0}(l, T)\right]
$$

where $\mathcal{S}_{0}(l, T)$ is the free-fermion entropy, and the large $l$ limit indicates the thermodynamic (macroscopic) limit; the zero-temperature limit is taken after the macroscopic limit. The large $l / \beta$ limit suggests that Eq. (I2) would be convenient to employ for the partition function, as only the lowest or ground state would need to be used from the summation. The impurity entropy at $T=0$ can then be shown to be $\mathcal{S}_{\text {imp }}(T=0)=\ln \langle A \mid 0\rangle\langle 0 \mid B\rangle$, where $|0\rangle$ denotes the ground state. This can be understood as the sum of entropies arising form the two boundaries $A, B[19,20]$. Applying the "fusion-rule" hypothesis of Affleck and Ludwig along with Cardy's equations (in conjunction with the Verlinde formula [23]) leads to Eq. (J3). Considering our maverick coset formulation, we focus only on the $\widetilde{\mathrm{SU}}(2)_{8}$ sector (taking $\eta=4$ canonical normalization) as the remaining sectors are decoupled from the impurity. This allows us to employ the modular S-matrix for canonically normalized $\mathrm{SU}(2)_{k}$,

$$
S_{n}^{m}(k)=\sqrt{\frac{2}{k+2}} \sin \left[\frac{\pi(2 m+1)(2 n+1)}{k+2}\right] .
$$

The residual entropy (for generalized $k$ channels) is thus

$$
\mathcal{S}_{\text {imp }}(T=0)=\ln \left(\frac{S_{1 / 2}^{0}(k)}{S_{0}^{0}(k)}\right)=\ln \left(\frac{\sin \frac{2 \pi}{k+2}}{\sin \frac{\pi}{k+2}}\right) .
$$

Taking $k=8$ in Eq. (J3) yields the residual entropy given in Sec. IX.

[1] S.-S. Lee, Recent Developments in Non-Fermi Liquid Theory, Annu. Rev. Condens. Matter Phys. 9, 227 (2018).

[2] G. R. Stewart, Non-Fermi-Liquid Behavior in $d$ - and f-Electron Metals, Rev. Mod. Phys. 73, 797 (2001).

[3] Jun Kondo, Resistance Minimum in Dilute Magnetic Alloys, Prog. Theor. Phys. 32, 37 (1964).

[4] A. A. Abrikosov, Electron Scattering on Magnetic Impurities in Metals and Anomalous Resistivity Effects, Physics Physique Fizika 2, 5 (1965).
[5] P. W. Anderson, A Poor Man's Derivation of Scaling Laws for the Kondo Problem, J. Phys. C 3, 2436 (1970).

[6] A. C. Hewson, The Kondo Problem to Heavy Fermions, Cambridge Studies in Magnetism (Cambridge University Press, Cambridge, England, 1993).

[7] P. Coleman, Theory Perspective: SCES 2016, Philos. Mag. 97, 3527 (2017).

[8] P. Nozières, A “Fermi-Liquid" Description of the Kondo Problem at Low Temperatures, J. Low Temp. Phys. 17, 31 (1974).

[9] K. G. Wilson, The Renormalization Group: Critical Phenomena and the Kondo Problem, Rev. Mod. Phys. 47, 773 (1975).

[10] N. Andrei, K. Furuya, and J. H. Lowenstein, Solution of the Kondo Problem, Rev. Mod. Phys. 55, 331 (1983).

[11] N. Andrei, Diagonalization of the Kondo Hamiltonian, Phys. Rev. Lett. 45, 379 (1980).

[12] I. Affleck, A Current Algebra Approach to the Kondo Effect, Nucl. Phys. B336, 517 (1990).

[13] N. Andrei and C. Destri, Solution of the Multichannel Kondo Problem, Phys. Rev. Lett. 52, 364 (1984).

[14] A. M. Tsvelick and P. B. Wiegmann, Exact Solution of the Multichannel Kondo Problem, Scaling, and Integrability, J. Stat. Phys. 38, 125 (1985).

[15] A. M. Tsvelick and P. B. Wiegmann, Solution of the n-Channel Kondo Problem (Scaling and Integrability), Z. Phys. B 54, 201 (1984).

[16] I. Affleck and A. W. W. Ludwig, Critical Theory of Overscreened Kondo Fixed Points, Nucl. Phys. B360, 641 (1991).

[17] I. Affleck and A. W. W. Ludwig, The Kondo Effect, Conformal Field Theory and Fusion Rules, Nucl. Phys. B352, 849 (1991).

[18] A. W. W. Ludwig and I. Affleck, Exact, Asymptotic, Three-Dimensional, Space- and Time-Dependent, Green's Functions in the Multichannel Kondo Effect, Phys. Rev. Lett. 67, 3160 (1991).

[19] I. Affleck and A. W. W. Ludwig, Exact Conformal-FieldTheory Results on the Multichannel Kondo Effect: SingleFermion Green's Function, Self-Energy, and Resistivity, Phys. Rev. B 48, 7297 (1993).

[20] I. Affleck and A. W. W. Ludwig, Universal Noninteger “Ground-State Degeneracy” in Critical Quantum Systems, Phys. Rev. Lett. 67, 161 (1991).

[21] A.W.W. Ludwig, Exact Results on the Multi-Channel Kondo Effect from Conformal Field Theory, Physica B (Amsterdam) 199-200, 406 (1994).

[22] A. M. Sengupta and Y. Baek Kim, Overscreened Single-Channel Kondo Problem, Phys. Rev. B 54, 14918 (1996).

[23] I. Affleck, Conformal Field Theory Approach to the Kondo Effect, Acta Phys. Pol. B 26, 1869 (1995), https://www .actaphys.uj.edu.pl/fulltext?series $=$ Reg $\&$ vol=26\&page $=$ 1869.

[24] I. Affleck, Quantum Impurity Problems in Condensed Matter Physics, in Exact Methods in Low-Dimensional Statistical Physics and Quantum Computing (Oxford University, New York, 2008), Vol. 89, pp. 3-65.

[25] Y. Kuramoto, H. Kusunose, and A. Kiss, Multipole Orders and Fluctuations in Strongly Correlated Electron Systems, J. Phys. Soc. Jpn. 78, 072001 (2009). 
[26] J. A. Mydosh and P. M. Oppeneer, Colloquium: Hidden Order, Superconductivity, and Magnetism: The Unsolved Case of $\mathrm{URu}_{2} \mathrm{Si}_{2}$, Rev. Mod. Phys. 83, 1301 (2011).

[27] P. Santini, S. Carretta, G. Amoretti, R. Caciuffo, N. Magnani, and G. H. Lander, Multipolar Interactions in f-Electron Systems: The Paradigm of Actinide Dioxides, Rev. Mod. Phys. 81, 807 (2009).

[28] J. G. Rau and M. J. P. Gingras, Frustrated Quantum RareEarth Pyrochlores, Annu. Rev. Condens. Matter Phys. 10, 357 (2019).

[29] E. W. Rosenberg, J.-H. Chu, J. P. C. Ruff, A. T. Hristov, and I. R. Fisher, Divergence of the Quadrupole-Strain Susceptibility of the Electronic Nematic System $\mathrm{YbRu}_{2} \mathrm{Ge}_{2}$, Proc. Natl. Acad. Sci. U.S.A. 116, 7232 (2019).

[30] D. L. Cox, Quadrupolar Kondo Effect in Uranium Heavy-Electron Materials?, Phys. Rev. Lett. 59, 1240 (1987).

[31] D. L. Cox and A. Zawadowski, Exotic Kondo Effects in Metals: Magnetic Ions in a Crystalline Electric Field and Tunnelling Centres, Adv. Phys. 47, 599 (1998).

[32] D. L. Cox, The Quadrupolar Kondo Effect: A New Mechanism for Heavy Electrons, J. Magn. Magn. Mater. 76-77, 53-58 (1988).

[33] A. S. Patri, I. Khait, and Y. B. Kim, Emergent Non-FermiLiquid Phenomena in Multipolar Quantum Impurity Systems, Phys. Rev. Research 2, 013257 (2020).

[34] J. Gan, N. Andrei, and P. Coleman, Perturbative Approach to the Non-Fermi-Liquid Fixed Point of the Overscreened Kondo Problem, Phys. Rev. Lett. 70, 686 (1993).

[35] J Gan, On the Multichannel Kondo Model, J. Phys. Condens. Matter 6, 4547 (1994).

[36] P. Di Francesco, P. Mathieu, and D. Senechal, Conformal Field Theory, Graduate Texts in Contemporary Physics (Springer-Verlag, New York, 1997).

[37] J. L. Cardy, Conformal Invariance and Surface Critical Behavior, Nucl. Phys. B240, 514 (1984).

[38] J. L. Cardy, Effect of Boundary Conditions on the Operator Content of Two-Dimensional Conformally Invariant Theories, Nucl. Phys. B275, 200 (1986).

[39] T. Onimaru and H. Kusunose, Exotic Quadrupolar Phenomena in Non-Kramers Doublet Systems the Cases of $\operatorname{PrT}_{2} \mathrm{Zn}_{20}(\mathrm{~T}=\mathrm{Ir}, R h)$ and $\mathrm{PrT}_{2} \mathrm{Al}_{20}(\mathrm{~T}=\mathrm{V}, T i)$, J. Phys. Soc. Jpn. 85, 082002 (2016).

[40] M. Tsujimoto, Y. Matsumoto, T. Tomita, A. Sakai, and S. Nakatsuji, Heavy-Fermion Superconductivity in the Quadrupole Ordered State of $\operatorname{PrV}_{2} \mathrm{Al}_{20}$, Phys. Rev. Lett. 113, 267001 (2014).

[41] K. Hattori and H. Tsunetsugu, Antiferro Quadrupole Orders in Non-Kramers Doublet Systems, J. Phys. Soc. Jpn. 83, 034709 (2014).

[42] S. Lee, S. Trebst, Y. B. Kim, and A. Paramekanti, Landau Theory of Multipolar Orders in $\operatorname{Pr}(\mathrm{Y})_{2} \mathrm{X}_{20}$ Kondo Materials (Y = Ti, V, Rh, Ir; X = Al, Zn), Phys. Rev. B 98, 134447 (2018).

[43] A. S. Patri, A. Sakai, S. Lee, A. Paramekanti, S. Nakatsuji, and Y. Baek Kim, Unveiling Hidden Multipolar Orders with Magnetostriction, Nat. Commun. 10, 4092 (2019).
[44] A. Sakai and S. Nakatsuji, Kondo Effects and Multipolar Order in the Cubic $\operatorname{PrTr}_{2} \mathrm{Al}_{20}(\mathrm{Tr}=\mathrm{Ti}, V)$, J. Phys. Soc. Jpn. 80, 063701 (2011).

[45] T. Onimaru, K. Izawa, K. T. Matsumoto, T. Yoshida, Y. Machida, T. Ikeura, K. Wakiya, K. Umeo, S. Kittaka, K. Araki, T. Sakakibara, and T. Takabatake, QuadrupoleDriven Non-Fermi-Liquid and Magnetic-Field-Induced Heavy Fermion States in a Non-Kramers Doublet System, Phys. Rev. B 94, 075134 (2016).

[46] M. Fu, A. Sakai, N. Sogabe, M. Tsujimoto, Y. Matsumoto, and S. Nakatsuji, Unveiling Quadrupolar Kondo Effect in the Heavy Fermion Superconductor prv2al20, J. Phys. Soc. Jpn. 89, 013704 (2020).

[47] P. Nozières and A. Blandin, Kondo Effect in Real Metals, J. Phys France 41, 193 (1980), https://hal.archives-ouvertes.fr/ jpa-00209235/.

[48] S. Nagashima, T. Nishiwaki, A. Otani, M. Sakoda, E. Matsuoka, H. Harima, and H. Sugawara, De Haas-van Alphen Effect in $\mathrm{RTi}_{2} \mathrm{Al}_{20}(\mathrm{R}=\mathrm{La}, P r$, and $S m)$, J. Phys. Soc. Jpn. Conf. Proc. 3, 011019 (2014).

[49] T. Kubo, E. Matsuoka, H. Kotegawa, H. Tou, A. Nakamura, D. Aoki, H. Harima, and H. Sugawara, Fermi Surface of the Heavy-Fermion Superconductor $\operatorname{PrTi}_{2} \mathrm{Al}_{20}$, J. Phys. Soc. Jpn. 89, 084704 (2020).

[50] P. Ginsparg, Applied Conformal Field Theory, arXiv:hep-th/ 9108028.

[51] D. C. Dunbar and K. G. Joshi, Characters for Coset Conformal Field Theories and Maverick Examples, Int. J. Mod. Phys. A 08, 4103 (1993).

[52] D. C. Dunbar and K. G. Joshi, Maverick Examples of Coset Conformal Field Theories, Mod. Phys. Lett. A 08, 2803 (1993).

[53] K. Kaneko, N. Metoki, R. Shiina, T. D. Matsuda, M. Kohgi, K. Kuwahara, and N. Bernhoeft, Neutron Scattering Study on the Field-Induced $O_{x y}$-Type Antiferroquadrupolar Ordering of the Heavy-Fermion Superconductor $\mathrm{PrOs}_{4} \mathrm{Sb}_{12}$, Phys. Rev. B 75, 094408 (2007).

[54] G. Friemel, Y. Li, A. V. Dukhnenko, N. Y. Shitsevalova, N. E. Sluchanko, A. Ivanov, V. B. Filipov, B. Keimer, and D.S. Inosov, Resonant Magnetic Exciton Mode in the Heavy-Fermion Antiferromagnet $\mathrm{CeB}_{6}$, Nat. Commun. 3, 830 (2012).

[55] M. Ye, H.-H. Kung, P. F. S. Rosa, E. D. Bauer, Z. Fisk, and G. Blumberg, Raman Spectroscopy of $f$-Electron Metals: An example of $\mathrm{CeB}_{6}$, Phys. Rev. Mater. 3, 065003 (2019).

[56] P. Thalmeier, A. Akbari, and R. Shiina, Multipolar Order and Excitations in Rare-Earth Boride Kondo Systems, arXiv:1907.10967.

[57] P. Chandra, P. Coleman, J. A. Mydosh, and V. Tripathi, Hidden Orbital Order in the Heavy Fermion Metal $\mathrm{URu}_{2} \mathrm{Si}_{2}$, Nature (London) 417, 831 (2002).

[58] V. Tripathi, P. Chandra, and P. Coleman, Itinerancy and Hidden Order in $\mathrm{URu}_{2} \mathrm{Si}_{2}$, J. Phys. Condens. Matter 17, 5285 (2005).

[59] K. Haule and G. Kotliar, Arrested Kondo Effect and Hidden Order in $\mathrm{URu}_{2} \mathrm{Si}_{2}$, Nat. Phys. 5, 796 (2009).

[60] M. E. Pezzoli, M. J. Graf, K. Haule, G. Kotliar, and A. V. Balatsky, Local Suppression of the Hidden-Order 
Phase by Impurities in $\mathrm{URu}_{2} \mathrm{Si}_{2}$, Phys. Rev. B 83, 235106 (2011).

[61] P. Chandra, P. Coleman, and R. Flint, Hastatic Order in the Heavy-Fermion Compound $\mathrm{URu}_{2} \mathrm{Si}_{2}$, Nature (London) 493, 621 (2013).

[62] H.-H. Lai, E. M. Nica, W.-J. Hu, S.-S. Gong, S. Paschen, and Q. Si, Kondo Destruction and Multipolar OrderImplications for Heavy Fermion Quantum Criticality, arXiv: 1807.09258.
[63] G. Zhang, J. S. Van Dyke, and R. Flint, Cubic Hastatic Order in the Two-Channel Kondo-Heisenberg Model, Phys. Rev. B 98, 235143 (2018).

[64] A. W. W. Ludwig, Methods of Conformal Field Theory in Condensed Matter Physics, in Low-Dimensional Quantum Field Theories for Condensed Matter Physicists (World Scientific, 1995), pp. 389-455, https://doi.org/10.1142/ 9789814447027_0007. 